\title{
Sixteen weeks of aerobic training influence spirometry, s-klotho, and hemodynamic responses to sub-maximal exercise testing in obese men with severe obstructive sleep apnea
}

\begin{abstract}
Purpose: The purpose of the study was to examine the influences of sixteen weeks of aerobic training on s-klotho levels, hemodynamic responses, and spirometry variables in obese men with severe obstructive sleep apnea before, during, and after the Six Minute Walk Test.

Methods: fifty men, age $57.6 \pm 6.82$ years, volunteered to participate in this study. Twenty-five obese subjects and diagnosed with severe OSA underwent sixteen weeks of aerobic exercise. Twenty-five healthy, untrained, age and sex-matching subjects were assigned to the control group. S-klotho levels, hemodynamic responses, and spirometry related values were obtained at various timings before, during, and after the Six Minute Walk Test (6MWT). Repeated measures were obtained upon enrollment, after eight weeks of exercise, and after sixteen weeks of exercise.
\end{abstract}

Results: Significant effects of time, exercise, and the interaction of timexexercise were noted for s-klotho concentrations and the vast majority of other variables.

Conclusions: sixteen weeks of aerobic training have a positive effect on s-klotho levels, hemodynamic responses at rest, during, and after the Six Minute Walk Test, as well as on spirometry in men with severe OSA.

Keywords: severe OSA, FEV1, FVC, FEV1/FVC ratio, S-klotho, 6MWT, hemodynamic responses, spirometry, blood pressure, lactate, heart rate
Volume 2 Issue 4 - 2018

\author{
Saghiv M,' Manisha Sawhney, ${ }^{2}$ Lark Welch, ${ }^{3}$ \\ Ben-Sira D, ${ }^{4}$ Goldhammer E, Sagiv $M^{4}$ \\ 'Department of Human Performance and Leisure Studies, \\ North Carolina A \& T State University, USA \\ 2Department of Psychology, University of Mary, USA \\ ${ }^{3}$ Department of Kinesiology, University of Mary, USA \\ ${ }^{4}$ Department of Life Sciences, The Zinman College of Physical \\ Education at the Wingate Collage, Israel \\ ${ }^{5}$ Heart Institute Bnai-Zion Haifa Medical Center, Israel
}

Correspondence: Moran S Saghiv, Associate Professor of Clinical Exercise Physiology, Department of Exercise Physiology, Casey Center, Room I4IB, University of Mary,7500 University Drive, Bismarck, ND 58504, USA, Tel 70I3558103, Fax 70I2557687,Email mssaghiv@umary.edu

Received: March 08, 2018 | Published: July 30,2018

\section{Introduction}

Klotho is an enzyme that in humans is encoded by the KL gene. S-klotho is a type I membrane protein first documented in $1997 .{ }^{2}$ The protein is associated with the degenerative process, acceleration and/or deceleration of aging, bone lose, and alcohol consumption..$^{3-7}$ Furthermore, s-klotho has a role in determining the sensitivity to Insulin, and mediation of the binding of FGF19, FGF20, and FGR23 to their receptors as part of the growth process at the cellular level. ${ }^{6}$

In addition, S-klotho is suspected of having cardiovascular protecting properties via means of endothelium-derived NO production. ${ }^{8,9}$ S-klotho affects cellular Calcium homeostasis via increased expression of TRPV5, decreased TRPC6, and increases membrane expression of Inward Rectifier ROMK. In mice, underexpression causes Hyper-vitaminosis of Vitamin D, altered mineralion homeostasis resulting in accelerated aging, a syndrome of accelerated aging, arteriosclerosis, impaired endothelium-dependent vasodilation, and impaired angiogenesis. ${ }^{6,10-13}$

Over-expression may prolong life span by $19-31 \%$ (in mice) In humans, exercise modalities influence klotho gene expression epigenetically and positively leading to increased expression. ${ }^{14}$ Prior studies show improvements in work capacity and performance alike. ${ }^{15,16}$

Sleep apnea (SA) is a respiratory disorder that occurs at night with symptoms that may be present during daytime as well. ${ }^{17}$ Three forms of sleep apnea exist, including Obstructive Sleep Apnea (OSA), Central Sleep Apnea (CSA), and Mixed Sleep Apnea (MSA). ${ }^{17}$ OSA is the most common form of SA and is due to blockage of airflow within the respiratory system. ${ }^{17}$ OSA is twice as much common in men, affecting up to $6 \%$ of adults, most commonly aged 55-60years. OSA may occur in children, though its prevalence is most definitely lower in children. ${ }^{18}$

The leading risk factors for SA include being of male sex, obesity, above age forty, neck diameter of 16-17 inches or greater $(40.64-43.18 \mathrm{~cm})$, enlarged tonsils or tongue, small jaw bone, gastroesophageal reflux, a variety of allergies, Sinusitis, a family history, and a deviated septum..$^{19,20}$ OSA is associated with reduced functional capacity. ${ }^{18-20}$ On the other hand, aerobic exercise improves cardiorespiratory aspects of function in people with OSA. ${ }^{21-25}$

The six Minute Walk Test (6MWT) is an exercise test that may be sub-maximal and/or maximal according to extent of effort on the participant's behalf. As part of the 6MWT the participant is instructed to achieve the greatest distance possible within 6minutes of walking. ${ }^{26}$ During the test, it is important to abstain from influencing the performance of the participant in order for the results and process to be genuine and free of external affecters. ${ }^{27}$

The overall distance achieved and the comparison to prior results influence future prognosis. ${ }^{28}$ While correlations to positive vs negative prognosis exist regarding the results of the 6MWT, interpretation within the actual distances is complex and perhaps ill-advised. ${ }^{29}$

Spirometry related variables such as FEV1 predicted (\%), FVC predicted (\%), and FEV1/FVC ratio (also known as the TiffeneauPinelli index) indicate the existence, or lack of, conductance and/or obstructive abnormalities in the respiratory system. ${ }^{30}$ People with diagnosed OSA commonly manifest spirometry values of negative 
implications to function and health. ${ }^{31}$ Such values are associated with poor exercise tolerance, decreased aerobic power (maximal oxygen uptake; $\mathrm{VO}_{2} \max$ ), and a shorter lifespan (years). ${ }^{32}$

To the best of the authors' knowledge, no prior published data exists pertaining the influences of aerobic exercise on s-klotho in men with OSA. Thus, the aim of this study was to investigate the influences of sixteen weeks of aerobic training on s-klotho $\left(\mathrm{pg} \cdot \mathrm{mL}^{-1}\right)$, heart rate $(\mathrm{bpm})$, blood pressure $(\mathrm{mmHg})$, Lactate $\left(\mathrm{mmol} \cdot \mathrm{L}^{-1}\right)$, Oxygen saturation $\left(\mathrm{O}_{2}\right.$ sat, \%), rate of perceived exertion (RPE, Scale), FEV1 predicted (\%), FVC predicted (\%),FEV1/FVC ratio, and the occurrence of angina and/or dyspnea event before and after a 6MWT in men with severe OSA.

\section{Methods}

After achieving IRB approval to conduct this study, twenty-five obese men clinically diagnosed by their physician with severe OSA $(\mathrm{BMI}>30 ; \mathrm{AHI}>30 / \mathrm{h})$ volunteered to participate in this study and have complied with all the demands of it. Subjects enrolled within 4weeks of their initial diagnosis and were untrained at the beginning of the study.

Twenty-five healthy, untrained, age and sex matching subjects volunteered and were assigned to the control group. Candidates were excluded from participating as control subjects if they presented with any clinical symptoms (physical and mental), were trained, and/or were medicated for more than hypertension. Control group subjects visited with the researchers only once. Subjects diagnosed with OSA visited with the researchers three times in total (enrollment; after eight weeks of exercise; after sixteen weeks of exercise weeks). Subjects were instructed to completely avoid alcohol consumption of any sort, exercise of any sort within twenty-four hours of data collection, caffeine, use of any medications other than subscribe by their physician (OSA diagnosed only), and/or stressful situations at least eight hours prior to their data collection sessions. All subjects self-reported their compliance with these instructions prior to each session.

The first visit was dedicated to signing the informed consent form (if not achieved before), risk stratification according to health history questionnaire (HHQ), inclusion or exclusion from the study, and obtaining baseline measures. Severe OSA diagnosed prospective subjects were excluded if they had diagnosed CAD, PAD, CVD, Diabetes, COPD, overlap syndrome, were post-surgery of any kind, had Cancer, were treated for Cancer, had a mental health condition, were medicated for other health conditions other than hypertension and/or OSA, and/or were not cleared for exercise by their physician. All included subjects were authorized by their physician to abstain from oxygen supplementation of any kind for the duration of the study.

All subjects reported to the lab between 6-8am. Upon arrival, subjects fulfilled a short questionnaire pertaining to their compliance with the pre-data collection instructions and changes to physical and/or mental health. Subjects compliant and asymptomatic were then weighed and seated for five minutes. Following the collection of baseline values (Table 1), the subjects underwent the 6MWT. Researcher have prepared in advance a 30-meter stretch of unimpeded walkway and have positioned three chairs along the way (at every end, and in the middle).

Subjects were instructed to achieve the greatest distance possible within six minutes and were instructed to inform the researchers immediately if they could not continue, had to sit down, or felt bad. Subjects were informed that they are allowed to terminate the test at any time.

Table I Timing of obtaining measures before, during, and after the six minute walk test

\begin{tabular}{|c|c|c|c|c|c|c|c|c|}
\hline Variable & Rest & +2 & +4 & IP & +5 & +10 & +15 & +45 \\
\hline Weight (kg) & yes & & & & & & & \\
\hline $\begin{array}{l}\text { Height } \\
(\mathrm{m})^{*}\end{array}$ & yes & & & & & & & \\
\hline $\begin{array}{l}\text { BMI }\left(\mathrm{Kg} \cdot \mathrm{m}^{-}\right. \\
\left.{ }^{2}\right)^{\dagger}\end{array}$ & yes & & & & & & & \\
\hline $\begin{array}{l}\text { Neckb } \\
(\mathrm{cm})\end{array}$ & yes & & & & & & & \\
\hline $\mathrm{AHI}(\mathrm{E} / \mathrm{hr})^{\dagger}$ & yes & & & & & & & \\
\hline $\begin{array}{l}\text { S-klotho } \\
\left(\mathrm{pg} \cdot \mathrm{mL}^{-1}\right)\end{array}$ & yes & & & yes & & & yes & yes \\
\hline HR (bpm) & yes & yes & yes & yes & yes & yes & yes & yes \\
\hline $\mathrm{BP}(\mathrm{mmHg})$ & yes & & & yes & yes & yes & yes & yes \\
\hline $\begin{array}{l}\text { FPG } \\
\left(\mathrm{mg} \cdot \mathrm{dL}^{-1}\right)\end{array}$ & yes & & & yes & yes & yes & yes & yes \\
\hline $\begin{array}{l}\text { LA } \\
\left(\mathrm{mmol} \cdot \mathrm{L}^{-1}\right)\end{array}$ & yes & & & yes & yes & yes & yes & yes \\
\hline Angina $^{\ddagger}$ & yes & yes & yes & yes & yes & yes & yes & yes \\
\hline Dyspnea $^{\ddagger}$ & yes & yes & yes & yes & yes & yes & yes & yes \\
\hline $\mathrm{RPE}^{\ddagger}$ & yes & yes & yes & yes & yes & yes & yes & yes \\
\hline O2sat (\%) & yes & yes & yes & yes & yes & yes & yes & yes \\
\hline FEVI (L) & yes & & & yes & & & & \\
\hline FVC (L) & yes & & & yes & & & & \\
\hline $\mathrm{FEVI} \mathrm{FVC}^{\dagger}$ & yes & & & yes & & & & \\
\hline
\end{tabular}

m, meter; Kg, kilogram; BMI, body mass index; SBP, systolic blood pressure; $\mathrm{DBP}$, diastolic blood pressure; $\mathrm{HR}$, heart rate; $\mathrm{O} 2$ sat, oxygen saturation; $\mathrm{mmHg}$, millimeter of Mercury; dL, deciliter; mmol, millimole; mg, milligram; bpm, beats per minute; pg, picogram; $\mathrm{mL}$, milliliter; $\nmid$, calculated ratio; $\ddagger$, scale; FPG, fasting plasma glucose; LA, lactate; b, circumference; AHI, apnea hypopnea index; hr hour; E, event of hypopnea; RPE, rate of perceived exertion; FEVI, forced expiratory volume; FVC, forced vital capacity; *, measured only once during first visit and assumed as of no change throughout the study

Excluding the need for informed consent and HHQ, the protocol detailed in visit 1 , was the same for all visits pertaining to subjects with OSA.

In between visits to the lab, subjects with OSA were instructed to exercise three times per week at a target heart rate of $95-105 \mathrm{bpm}$ ( $\approx 60 \%$ of age-estimated maximal heart rate); according to the formula of $0.6 \times(220$-age $)$. During the first week, the duration of every exercise session was 20 minutes; session duration was increased by 30 seconds from week to week. Thus, during the second week of the study, every session was 20.5 minutes long; 21 minutes long during the sessions of the third week, etc. Heart rate range was kept constant throughout the study, causing the subjects to move faster if needed in order to stay within that range.

All blood samples were obtained according to universal precautions for blood borne pathogens from the Median Cubital Vein and stored in BD Vacutainer ${ }^{\mathrm{TM}}$ Venous Blood Collection Tubes: SSTTM Serum Separation Tubes with Conventional Stopper until analyzed; samples were immediately refrigerated. 
Blood samples were analyzed via Soluble Klotho (Human serum) ELISA Kit SK00708-08 (Adipo Bioscience, Inc.), with a Standard range of 313-20,000 $\mathrm{pg} \cdot \mathrm{mL}^{-1}$, Sensitivity of $80 \mathrm{pg} \cdot \mathrm{mL}^{-1}$; Intra-CV of $4-6 \%$; and Inter-CV of 8-10\%. Blood pressure was obtained utilizing an Omron sphygmomanometer; heart rate via FS2c Polar Heart Rate Monitor and strap, Oxygen saturation was obtained via KNIGHTROOXIMETER 5.2 Bluetooth Fingertip Pulse Oximeter; Lactate and fasting plasma glucose $\left(\mathrm{FPG} ; \mathrm{mg} \cdot \mathrm{dL}^{-1}\right)$ were obtained via Point-ofCare testing using a Lactate "Scout" Analyzer (Basic) B7023-255 and a Roche Diagnostics CoaguChek XS Professional Meter Kit respectively. The treadmill used in this study was a Bowflex Results Series $^{\mathrm{TM}}$ BXT216 Treadmill. Spirometry was conducted via a MIR Spirobank II Smart BLE spirometer. A charged AED was present in the lab at all times, as well as a landline phone, and backup cellphone. During all data collection sessions, at least one of the researchers was ACLS certified.

\section{Statistical analysis}

A two way ANOVA with repeated measures was utilized to analyze the data obtained. Data is presented as mean and standard deviation when appropriate with significance levels ( $\mathrm{P}$ value).

\section{Results}

\section{Overview, enrollment, and descriptive data}

All subjects completed this study without any prolonged adverse reactions. Some subjects had a temporary reaction to their blood being drawn (dizziness, elevated heart rate, hypertension, hypotension, and in two cases subjects reacted with syncope). Nine different subjects had acute adverse reactions to the Six Minute Walk Test including shortness of breath, Angina, and lightheadedness on several occasions. All subjects left the lab without any adverse clinical signs other than pre-existing due to their OSA.

Control group were insignificantly older and shorter $(p>0.05)$. Experimental group's weight and BMI were significantly higher $(p \leq 0.05)$. Neck circumference and AHI were significantly higher for the experimental group $(\mathrm{p} \leq 0.01)$.

Weight, BMI, and Neck circumference were insignificantly lower for the experimental group at the end of the study ( $>00.05)$. AHI decreased significantly by the end of the study $(\mathrm{p} \leq 0.01)$. Relevant data are presented in Table 2.

Table 2 Resting $\dagger$ descriptive data and changes in obtained measurements, according to visits. (Mean \pm SD)

\begin{tabular}{|c|c|c|c|c|}
\hline Variable & $\begin{array}{l}\text { Enroll } \\
\text { healthy }\end{array}$ & Enroll OSA & EXS+8Wk & EXS+16Wk \\
\hline \multicolumn{2}{|c|}{ No intervention } & \multicolumn{3}{|c|}{ Exercise intervention } \\
\hline $\mathbf{N}$ & 25 & 25 & 25 & 25 \\
\hline Age (years) & $58.5 I \pm 3.6$ & $56.25 \pm 4.8$ & $*$ & $*$ \\
\hline Height (m) & $1.75 \pm 3.6$ & $1.77 \pm 2.1$ & $*$ & * \\
\hline Height (in) & $68.89 \pm 1.41$ & $69.68 \pm 0.82$ & * & * \\
\hline Weight (Kg) & $81.73 \pm 4.3$ & $1 \mid 6.27 \pm 5.1$ & || $3.8 \mid \pm 4.2$ & $111.56 \pm 5.5$ \\
\hline Weight (lb) & $179.8 \pm 9.46$ & $255.2 \pm 11.22$ & $250.38 \pm 9.24$ & $245.43 \pm 12.1$ \\
\hline BMI $\left(\mathrm{Kg} \cdot \mathrm{m}^{-2}\right)$ & $26.71 \pm 0.59$ & $37.15 \pm 1.98$ & $36.33 \pm 1.25$ & $35.61 \pm 1.34$ \\
\hline $\operatorname{Neck}^{\mathrm{b}}(\mathrm{cm})$ & $35.21 \pm 2.59$ & $42.15 \pm 4.48$ & $40.33 \pm 4.18$ & $40.17 \pm 4.28$ \\
\hline $\mathrm{AHI}(\mathrm{E} / \mathrm{hr})$ & $3.01 \pm 0.53$ & $45.45 \pm 14.48$ & $41.7 \pm 17.48$ & $40.15 \pm 15.3$ \\
\hline
\end{tabular}

$\mathrm{N}$, number of subjects; m, meter; in, inch; Kg, kilogram; lb, pound; BMI, body mass index; SD, standard deviation; *, variable values assumed the same as in baseline;b, circumference; $\mathrm{AHI}$, apnea hypopnea index; hr, hour; E, event of hypopnea; EXS, exercise; Wk, weeks;

The design of this study includes a vast number of variables, with a very high number of possible comparisons. In order to avoid over burdening the reader as much as possible, the significance of the comparisons are presented in essence only.

\section{S-klotho (pg mL-I)}

All possible comparisons within and between groups were significant $(\mathrm{p} \leq 0.05)$. All data regarded s-klotho are presented in Table 3.

Table 3 s-klotho concentrations before and after the Six Minute Walk Test according to visit and timing of measurement (Mean \pm SD)

\begin{tabular}{|c|c|c|c|c|}
\hline $\begin{array}{l}\text { S-klotho } \\
\text { (pg mL-1) }\end{array}$ & $\begin{array}{l}\text { Enroll } \\
\text { healthy }\end{array}$ & $\begin{array}{l}\text { Enroll } \\
\text { OSA }\end{array}$ & EXS+8Wk & EXS+16Wk \\
\hline \multicolumn{5}{|c|}{ Exercise intervention } \\
\hline $\mathbf{N}$ & 25 & 25 & 25 & 25 \\
\hline $\operatorname{Rest}^{\dagger}$ & $475.5 \pm 102$ & $262.4 \pm 58$ & $323.6 \pm 52$ & $371.45 \pm 62$ \\
\hline $\begin{array}{l}\text { Immediate- } \\
\text { post }\end{array}$ & $677.3 \pm 98$ & $342.64 \pm 88$ & $416.18 \pm 52$ & $462.05 \pm 55$ \\
\hline $15 \mathrm{~min}$ post & $637.4 \pm 88$ & $292.3 \pm 81$ & $351.48 \pm 62$ & $409.33 \pm 75$ \\
\hline $45 \mathrm{~min}$ post & $554.6 I \pm 97$ & $272.3 \pm 85$ & $336.48 \pm 43$ & $402.0 \pm 65$ \\
\hline
\end{tabular}

$\mathrm{N}$, number of subjects; pg, picogram; $\mathrm{mL}$, milliliter; SD, standard deviation; $\dagger$. values obtained while sitting; EXS, exercise; Wk, weeks

\section{Heart rate (HR; bpm)}

a. Rest to 45 minutes post (control)- Differences between rest, 10 minutes post, 15 minutes post, 45 minutes post, and between immediate-post and 2 minutes within were insignificant $(\mathrm{p}>0.05)$.

b. Rest to 45minutes post (experimental; Enrollment) Differences between rest, 15 minutes post, and 45 minutes post were insignificant $(\mathrm{p}>0.05)$.

c. Rest to 45 minutes post (experimental; +8 weeks) - Differences between rest, 15 minutes post, 45 minutes post, and between 2 and 4 minutes within were insignificant $(\mathrm{p}>0.05)$.

d. Rest to 45 minutes post (experimental; +16 weeks) - Differences between rest, 15 minutes post, 45 minutes post, and between 2 minutes and 4 minutes within were insignificant ( $p>0.05$ ).

e. Rest (between groups)- All comparisons were significantly lower for the control group, excluding after 16 weeks of exercise $(\mathrm{p} \leq 0.05)$.

f. Rest (experimental)- HR was significantly higher while untrained compared to while trained $(\mathrm{p} \leq 0.05)$. Differences while trained were insignificant $(\mathrm{p}>0.05)$.

g. 2minutes within (between groups) - All comparisons were significant $(\mathrm{p} \leq 0.05)$.

h. 2minutes within (experimental) - HR upon enrollment was significantly higher compared to all other sessions $(\mathrm{p} \leq 0.05)$. All other comparisons were insignificant $(\mathrm{p}>0.05)$.

i. 4minutes within (between groups) - All comparisons were significant $(\mathrm{p} \leq 0.05)$. 
j. 4minutes within (experimental) - All comparisons were insignificant $(\mathrm{p}>0.05)$.

k. Immediate-post (between groups) - The comparison upon enrollment was insignificant $(\mathrm{p}>0.05)$. All other comparisons were significant $(\mathrm{p} \leq 0.05)$.

1. Immediate-post (experimental)- Comparison between enrollment and 8 weeks was significant $(\mathrm{p} \leq 0.05)$. All other comparisons were insignificant $(\mathrm{p}>0.05)$.

m. 5minutes post (between groups) - All comparisons were significant $(\mathrm{p} \leq 0.05)$.

n. 5minutes post (experimental)- Comparison of enrollment and 8 weeks of exercising was insignificant $(\mathrm{p}>0.05)$. All other comparisons were significant $(\mathrm{p} \leq 0.05)$.

o. 10minutes post (between groups) - All comparisons were significant $(\mathrm{p} \leq 0.01)$.

p. 10minutes post (experimental)- Comparison for while trained was insignificant $(p>0.05)$. All other comparisons were significant $(\mathrm{p} \leq 0.05)$.

q. 15minutes post (between groups)- Comparison to 16 weeks was insignificantly $(\mathrm{p}>0.05)$. All other comparisons were significant $(\mathrm{p} \leq 0.05)$

r. 15minutes post (experimental)-All other comparisons were significant $(\mathrm{p} \leq 0.05)$.

s. 45minutes post (between groups) - Comparison to 16 weeks insignificant $(\mathrm{p}>0.05)$. All other comparisons were significant $(\mathrm{p} \leq 0.05)$.

t. 45minutes post (experimental): Comparison for while trained was insignificant $(p>0.05)$. All other comparisons were significant $(\mathrm{p} \leq 0.01)$. All data regarding heart rate are presented in Table 4.

Table 4 heart rate before, during, and after the Six Minute Walk Test according to visit and timing of measurement (Mean \pm SD)

\begin{tabular}{|c|c|c|c|c|}
\hline \multirow{3}{*}{$\begin{array}{l}\text { HR } \\
\text { (bpm) } \\
\mathbf{N}\end{array}$} & $\begin{array}{l}\text { Enroll } \\
\text { healthy }\end{array}$ & $\begin{array}{l}\text { Enroll } \\
\text { OSA }\end{array}$ & EXS+8Wk & EXS+16Wk \\
\hline & \multicolumn{2}{|c|}{ No intervention } & \multicolumn{2}{|c|}{ Exercise intervention } \\
\hline & 25 & 25 & 25 & 25 \\
\hline $\operatorname{Rest}^{\dagger}$ & $76.6 \pm 5.3$ & $87.4 \pm 6.7$ & $81.63 \pm 3.11$ & $78.08 \pm 4.03$ \\
\hline$+2 \min$ & $158.3 \pm 6.5$ & $\mid 53.1 \pm 8.2$ & $|47.0 \pm 4.4|$ & $|46.25 \pm 5.8|$ \\
\hline$+4 \min$ & $150.6 \pm 7.12$ & $|40 \pm| \mid .8$ & $|43.0 \pm 2.9|$ & $144.65 \pm 6.23$ \\
\hline $\begin{array}{l}\text { Immediate- } \\
\text { post }\end{array}$ & $160.3 \pm 9.1$ & $|57.3| \pm 8.7$ & $|53.3| \pm 7.1 \mid$ & $156.0 \pm 7.4$ \\
\hline $5 \mathrm{~min}$ post & $92.44 \pm 6.81$ & $134.1 \pm 4.33$ & $133.04 \pm 4.19$ & $129.34 \pm 4.4$ \\
\hline $10 \mathrm{~min}$ post & $76.89 \pm 4.61$ & $115.34 \pm 3.17$ & $102.66 \pm 4.78$ & $101.08 \pm 3.26$ \\
\hline $15 \mathrm{~min}$ post & $76.54 \pm 5.4$ & $88.14 \pm 5.46$ & $82.06 \pm 3.48$ & $78.44 \pm 2.61$ \\
\hline $45 \mathrm{~min}$ post & $76.2 \pm 3.31$ & $87.16 \pm 2.23$ & $81.08 \pm 2.71$ & $78.66 \pm 1.92$ \\
\hline
\end{tabular}

$\mathrm{N}$, number of subjects; HR, heart rate; bpm, beats per minute; SD, standard deviation; $\nmid$, values obtained while sitting; EXS, exercise; Wk, weeks

\section{Systolic blood pressure (SBP; $\mathrm{mmHg}$ )}

a. Rest to 45minutes post (control) - Immediate-post was significantly higher than all other values $(\mathrm{p} \leq 0.05)$. fiveminutes post was significantly higher than rest, 10 minutes post, 15 minutes post, and 45 minutes post $(\mathrm{p} \leq 0.05)$. Differences between rest, 10 minutes post, 15 minutes post, and 45 minutes post were insignificant $(\mathrm{p}>0.05)$.

b. Rest to 45minutes post (experimental; Enrollment) Immediate-post was significantly higher than all other values $(p \leq 0.05)$. 5minutes post was significantly higher than all other comparisons excluding immediate-post $(\mathrm{p} \leq 0.05)$. 10minutes post was significantly higher compared to rest, 15 minutes post, and 45 minutes post $(\mathrm{p} \leq 0.05)$. All other comparisons were insignificant $(\mathrm{p}>0.05)$.

c. Rest to 45 minutes post (experimental; +8 weeks) - Immediatepost was significantly higher than all other values $(\mathrm{p} \leq 0.05)$. 5 minutes post was significantly higher than all other comparisons excluding immediate-post $(\mathrm{p} \leq 0.05)$. 10minutes post was significantly higher compared to rest, 15 minutes post, and 45 minutes post $(p \leq 0.05)$. 15 minutes post was insignificantly higher compared to rest ( $>0.05)$, and insignificantly lower compared to 45 minutes post ( $\mathrm{p}>0.05)$.

d. Rest to 45 minutes post (experimental; +16weeks) - Immediatepost was significantly higher than all other values $(\mathrm{p} \leq 0.05)$. 5minutes post was significantly higher than all other comparisons excluding immediate-post $(\mathrm{p} \leq 0.05)$. 10minutes post was significantly higher compared to rest, 15 minutes post, and 45 minutes post $(p \leq 0.01) .15$ minutes post was insignificantly higher compared to rest ( $>0.05)$, and insignificantly lower compared to 45 minutes post ( $\mathrm{p}>0.05$ ).

e. Rest (between groups) - Comparison to eight weeks was insignificant $(\mathrm{p}>0.05)$. All other comparisons were significant $(\mathrm{p} \leq 0.05)$.

f. Rest (experimental) - Comparison for while trained was insignificant $(p>0.05)$. All other comparisons were significant $(\mathrm{p} \leq 0.05)$.

g. Immediate-post (between groups) - Control group's value was significantly lower compared to eight and sixteen weeks $(\mathrm{p} \leq 0.05)$, and significantly higher enrollment $(\mathrm{p} \leq 0.05)$.

h. Immediate-post (experimental) - All comparisons were significant $(\mathrm{p} \leq 0.05)$.

i. 5minutes post (between groups) - All comparisons were significant $(\mathrm{p} \leq 0.05)$.

j. 5minutes post (experimental) - All comparisons were insignificant $(\mathrm{p}>0.05)$.

k. 10minutes post (between groups) - All comparisons were significant $(\mathrm{p} \leq 0.01)$.

1. 10minutes post (experimental) - All comparisons were significant $(\mathrm{p} \leq 0.05)$.

m. 15minutes post (between groups) - Comparison was insignificantly lower compared to while trained $(\mathrm{p}>0.05)$.

n. 15 minutes post (experimental) - Enrollment was significantly higher compared to all other values $(\mathrm{p} \leq 0.05)$. Difference while trained was insignificant $(\mathrm{p}>0.05)$.

o. 45minutes post (between groups) - All comparisons were significant $(\mathrm{p} \leq 0.05)$.

p. 45minutes post (experimental) - Comparison between enrollment and while exercised was significant $(p \leq 0.05)$. Comparison while exercised was insignificant $(p>0.05)$. All 
data regarding systolic blood pressure are presented in Table 5 .

Table 5 systolic blood before and after the Six Minute Walk Test pressure according to visit and timing of measurement (Mean \pm SD)

\begin{tabular}{lllll}
$\begin{array}{l}\text { SBP } \\
(\mathbf{m m H g})\end{array}$ & $\begin{array}{l}\text { Enroll } \\
\text { healthy }\end{array}$ & $\begin{array}{l}\text { Enroll } \\
\text { OSA }\end{array}$ & EXS+8Wk & EXS+16Wk \\
\hline $\mathbf{N}$ & $\mathbf{2 5}$ & $\mathbf{2 5}$ & $\mathbf{2 5}$ & $\mathbf{2 5}$ \\
\hline Rest $^{\dagger}$ & $125.1 \pm 1.6$ & $133.3 \pm 3.6$ & $126.5 \pm 2.0$ & $125.37 \pm 2.4 \mid$ \\
$\begin{array}{l}\text { Immediate- } \\
\text { post }\end{array}$ & $182.3 \pm 5.53$ & $175.61 \pm 7.32$ & $183.13 \pm 5.1$ & $187.0 \pm 9.11$ \\
5 min post & $141.6 \pm 4.73$ & $165.2 \pm 3.54$ & $166.45 \pm 5.78$ & $164.33 \pm 3.79$ \\
\hline 10min post & $126.11 \pm 2.01$ & $150.66 \pm 4.07$ & $141.33 \pm 4.24$ & $133.44 \pm 4.96$ \\
\hline 15 min post & $125.22 \pm 1.54$ & $134.42 \pm 3.37$ & $128.43 \pm 3.39$ & $126.37 \pm 4.14$ \\
45 min post & $125.13 \pm 1.64$ & $137.7 \pm 2.72$ & $130.0 \pm 2.03$ & $129.54 \pm 1.9$ \\
\hline
\end{tabular}

$\mathrm{N}$, number of subjects; SBP, systolic blood pressure; $\mathrm{mmHg}$, millimeter of Mercury; SD, standard deviation; $t$, values obtained while sitting; EXS, exercise; Wk, weeks

\section{Diastolic blood pressure (DBP; $\mathbf{m m H g}$ )}

a. Rest to 45 minutes post (control) - Immediate-post was significantly higher than all other values $(\mathrm{p} \leq 0.05)$. All other comparisons were insignificant $(\mathrm{P}>0.05)$.

b. Rest to 45minutes post (experimental; Enrollment) Immediate-post value was significantly higher than all other values $(p \leq 0.05)$. 5minutes post was significantly higher than all other possible comparisons excluding immediatepost $(\mathrm{p} \leq 0.05)$. Differences between rest, 10minutes post, 15 minutes post, and 45 minutes post were insignificant $(\mathrm{p}>0.05)$

c. Rest to 45 minutes post (experimental; +8weeks) Immediate-post value was significantly higher than all other values $(p \leq 0.05)$. 5minutes post was significantly higher than all other comparisons excluding immediate-post $(\mathrm{p} \leq 0.05)$ Differences between rest, 10minutes post, 15 minutes post, and 45 minutes post were insignificant $(\mathrm{p}>0.05)$.

d. Rest to 45 minutes post (experimental; +16weeks) Immediate-post value was significantly higher than all other values $(p \leq 0.05)$. All other comparisons were insignificant $(\mathrm{p}>0.05)$.

e. Rest (between groups) - Comparison to sixteen weeks was significant $(\mathrm{p} \leq 0.05)$. All other comparisons were insignificant $(\mathrm{p}>0.05)$.

f. Rest (experimental) - All comparisons were insignificant $(\mathrm{p}>0.05)$.

g. Immediate-post (between groups) - All comparisons were significant $(\mathrm{p} \leq 0.05)$.

h. Immediate-post (experimental) - All comparisons were significant $(\mathrm{p} \leq 0.05)$.

i. 5minutes post (between groups) - All comparisons were significant $(\mathrm{p} \leq 0.05)$ excluding sixteen weeks $(\mathrm{p}>0.05)$.

j. 5minutes post (experimental) - All possible comparisons were insignificant $(\mathrm{p}>0.05)$.

k. 10minutes post (between groups) - All other comparisons were insignificant $(\mathrm{p}>0.05)$.
1. 10minutes post (experimental) - All comparisons were insignificant $(\mathrm{p}>0.05)$

m. 15minutes post (between groups) - All comparisons insignificant $(\mathrm{p}>0.05)$.

n. 15minutes post (experimental) -All comparisons were insignificant $(\mathrm{p}>0.05)$.

o. 45minutes post (between groups) - All comparisons were insignificant $(\mathrm{p}>0.05)$

p. 45minutes post (experimental) - All comparisons were insignificant $(\mathrm{p}>0.05)$. All data regarding diastolic blood pressure are presented in Table 6.

Table 6 Diastolic blood before and after the Six Minute Walk Test pressure according to visit and timing of measurement (Mean \pm SD)

\begin{tabular}{|c|c|c|c|c|}
\hline $\begin{array}{l}\text { DBP } \\
(\mathrm{mmHg})\end{array}$ & $\begin{array}{l}\text { Enroll } \\
\text { healthy }\end{array}$ & Enroll OSA & EXS+8Wk & EXS+16Wk \\
\hline $\mathbf{N}$ & 25 & 25 & 25 & 25 \\
\hline $\operatorname{Rest}^{\dagger}$ & $83.1 \pm 13.6$ & $86.5 \pm 12.3$ & $84.1 \pm 8.9$ & $84.17 \pm 5.21$ \\
\hline $\begin{array}{l}\text { Immediate- } \\
\text { post }\end{array}$ & $91.6 \pm 4.7$ & $98.1 \pm 5.6$ & $94.45 \pm 4.9$ & $92.1 \pm 5.2$ \\
\hline $5 \mathrm{~min}$ post & $86.5 \pm 4.25$ & $98.31 \pm 8.2$ & $89.42 \pm 3.91$ & $84.27 \pm 2.2$ \\
\hline $10 \mathrm{~min}$ post & $83.11 \pm 7.2$ & $85.78 \pm 10.12$ & $84.0 \pm 9.91$ & $85.06 \pm 6.2$ \\
\hline $15 \mathrm{~min}$ post & $83.3 \pm 10.1$ & $86.11 \pm 7.55$ & $83.56 \pm 8.54$ & $84.77 \pm 5.64$ \\
\hline $45 \mathrm{~min}$ post & $83.98 \pm 9.5$ & $85.77 \pm 4.25$ & $84.4 \pm 8.2$ & $84.06 \pm 4.91$ \\
\hline
\end{tabular}

$\mathrm{N}$, number of subjects; $\mathrm{DBP}$, diastolic blood pressure; $\mathrm{mmHg}$, millimeter of Mercury; SD, standard deviation; $\uparrow$, values obtained while sitting; EXS, exercise; Wk, weeks

\section{Oxygen saturation (O2sat; \%)}

Rest to 45minutes post (control) - Multiple comparisons were insignificant $(\mathrm{p}>0.05)$ including rest vs 2 minutes; rest vs 4 minutes; rest vs 5 minutes post; rest vs 10 minutes post; rest vs 15 minutes post; rest vs 45 minutes post; 2 minutes vs 4 minutes; 2 minutes vs immediate-post; 2 minutes vs 5 minutes post; 2 minutes vs 10 minutes post; 4 minutes vs 5 minutes post; 4 minutes vs 10 minutes post; 4 minutes vs 15 minutes post; 4 minutes vs 45 minutes post; immediate-post vs 5 minutes post; 5 minutes post vs 10 minutes post; 10 minutes post vs 15 minutes post; 10 minutes post vs 45 minutes post; and 15 minutes post vs 45 minutes post. All other comparisons were significant $(\mathrm{p} \leq 0.05)$.

Rest to 45minutes post (experimental; Enrollment) - Multiple comparisons were insignificant ( $\mathrm{p}>0.05$ ) including rest vs 15 minutes post; rest vs 45 minutes post; 2 minutes vs 4minutes; 2 minutes vs 10 minutes post; 4 minutes vs 5 minutes post; immediate-post vs 5 minutes post; 15 minutes post vs 45 minutes post. All other comparisons were significant $(\mathrm{p} \leq 0.05)$.

Rest to 45minutes post (experimental; +8weeks) - Multiple comparisons were insignificant ( $\mathrm{p}>0.05)$ including rest vs 10 minutes post; rest vs 15 minutes post; rest vs 45 minutes post; 2 minutes vs 4 minutes; 2 minutes vs 5 minutes post; 5 minutes post vs 10 minutes post; 5 minutes post vs 15 minutes post; 5 minutes post vs 45 minutes post; 10 minutes post vs 15 minutes post; 10 minutes post vs 45 minutes post; and 15 minutes post vs 45 minutes post. All other comparisons were significant $(\mathrm{p} \leq 0.05)$.

Rest to 45minutes post (experimental; +16weeks) - Multiple 
comparisons were insignificant ( $\mathrm{p}>0.05$ ) including rest vs 10 minutes post; rest vs 15 minutes post; rest vs 45 minutes post; 2 minutes vs 4minutes; 2 minutes vs 5 minutes post; 4 minutes vs immediate-post; 10 minutes post vs 15 minutes post; 10 minutes post vs 45 minutes post; and 15 minutes post vs 45 minutes post. All other comparisons were significant $(\mathrm{p} \leq 0.05)$.

Rest (between groups) - All comparisons were significant $(\mathrm{p} \leq 0.05)$

Rest (experimental) - Comparisons while trained compared to untrained were significant $(\mathrm{p} \leq 0.05)$. All other comparisons were insignificant $(\mathrm{p}>0.05)$.

2minutes within (between groups) - All comparisons were significant $(\mathrm{p} \leq 0.05)$.

2minutes within (experimental) - Comparisons between enrollment and while trained were significant $(\mathrm{p} \leq 0.05)$. All other comparisons were insignificant $(\mathrm{p}>0.05)$.

4minutes within (between groups) - All comparisons were significant $(\mathrm{p} \leq 0.05)$.

4minutes within (experimental) - Comparisons between enrollment and while trained were significant $(\mathrm{p} \leq 0.05)$. All other comparisons were insignificant $(\mathrm{p}>0.05)$.

Immediate-post (between groups) - All comparisons were significant $(\mathrm{p} \leq 0.05)$.

Immediate-post (experimental) - All comparisons were significant $(\mathrm{p} \leq 0.05)$.

5minutes post (between groups) - All comparisons were significant $(\mathrm{p} \leq 0.05)$.

5minutes post (experimental) - All comparisons between while trained and untrained were significant $(\mathrm{p} \leq 0.05)$. All other comparisons were insignificant $(\mathrm{p}>0.05)$.

10minutes post (between groups) - All comparisons were significant $(\mathrm{p} \leq 0.05)$.

10minutes post (experimental) - All comparisons between while trained and untrained were significant $(\mathrm{p} \leq 0.05)$. All other comparisons were insignificant $(\mathrm{p}>0.05)$.

15 minutes post (between groups) -All comparisons were significant $(\mathrm{p} \leq 0.05)$.

15minutes post (experimental) - All comparisons between while trained and untrained were significant $(\mathrm{p} \leq 0.05)$. All other comparisons were insignificant $(\mathrm{p}>0.05)$.

45minutes post (between groups) - All comparisons were significant $(\mathrm{p} \leq 0.05)$.

45minutes post (experimental) - All comparisons between while trained and untrained was significant $(\mathrm{p} \leq 0.05)$. All other comparisons were insignificant $(\mathrm{p}>0.05)$. All data regarding oxygen saturation are presented in Table 7.

Table 7 Oxygen saturation before, during, and after the Six Minute Walk Test according to visit and timing of measurement (Mean \pm SD)

\begin{tabular}{|c|c|c|c|c|}
\hline $\mathrm{O}_{2}$ sat (\%) & $\begin{array}{l}\text { Enroll } \\
\text { healthy }\end{array}$ & $\begin{array}{l}\text { Enroll } \\
\text { OSA }\end{array}$ & EXS+8Wk & EXS+16Wk \\
\hline \multicolumn{2}{|c|}{ No intervention } & \multicolumn{2}{|c|}{ Exercise intervention } & \\
\hline $\mathbf{N}$ & 25 & 25 & 25 & 25 \\
\hline $\operatorname{Rest}^{\dagger}$ & $96.89 \pm 1.4$ & $88.5 \pm 9.6$ & $91.5 \pm 6.51$ & $92.65 \pm 6.12$ \\
\hline$+2 \min$ & $95.29 \pm 2.8$ & $85.12 \pm 7.72$ & $87.22 \pm 6.13$ & $88.0 \pm 5.5$ \\
\hline$+4 \min$ & $96.07 \pm 3.63$ & $84.62 \pm 6.89$ & $86.77 \pm 6.3$ & $87.3 \pm 5.01$ \\
\hline $\begin{array}{l}\text { Immediate- } \\
\text { post }\end{array}$ & $94.88 \pm 4.98$ & $82.5 \pm 8.7$ & $84.22 \pm 6.13$ & $86.6 \pm 7.3$ \\
\hline $5 \mathrm{~min}$ post & $95.91 \pm 2.64$ & $83.25 \pm 6.66$ & $88.57 \pm 8.43$ & $89.03 \pm 5.76$ \\
\hline $10 \mathrm{~min}$ post & $96.75 \pm 1.55$ & $86.37 \pm 5.4 I$ & $91.02 \pm 4.75$ & $92.3 \pm 6.5$ \\
\hline $15 \mathrm{~min}$ post & $97.04 \pm 1.47$ & $88.63 \pm 4.97$ & $91.76 \pm 5.86$ & $92.21 \pm 4.33$ \\
\hline $45 \min$ post & $97.49 \pm 2.01$ & $88.85 \pm 6.76$ & $91.36 \pm 5.33$ & $92.16 \pm 6.1$ \\
\hline
\end{tabular}

$\mathrm{N}$, number of subjects; $\mathrm{O}_{2}$ sat, oxygen saturation; SD, standard deviation; $\dagger$, values obtained while sitting; EXS, exercise; Wk, weeks

\section{Rate of perceived exertion (RPE; scale)}

Rest to 45minutes post (control) - Multiple comparisons were insignificant $(\mathrm{p}>0.05)$ including rest vs 10 minutes post; rest vs 15 minutes post; rest vs 45 minutes post; 4 minutes vs immediate-post; 10 minutes post vs 15 minutes post; 10 minutes post vs 45 minutes post; and 15 minutes post vs 45 minutes post. All other comparisons were significant $(\mathrm{p} \leq 0.05)$

Rest to 45minutes post (experimental; Enrollment) - Multiple comparisons were insignificant ( $\mathrm{p}>0.05$ ) including rest vs 45 minutes post; 2 minutes vs 4 minutes; 2 minutes vs 5 minutes post; and 4 minutes vs 5 minutes post. All other comparisons were significant ( $\mathrm{p} \leq 0.05)$.

Rest to 45minutes post (experimental; +8weeks) - Multiple comparisons were insignificant ( $\mathrm{p}>0.05)$ including rest vs 15 minutes post; rest vs 45 minutes post; 2 minutes vs 4 minutes; and 15 minutes post vs forty-five minutes post. All other comparisons were significant $(\mathrm{p} \leq 0.05)$.

Rest to 45minutes post (experimental; +16weeks) - Multiple comparisons were insignificant ( $\mathrm{p}>0.05$ ) including rest vs 15 minutes post; rest vs 45 minutes post; 2 minutes vs 4 minutes; 2 minutes vs 5 minutes post; 4 minutes vs 5 minutes post; and 15 minutes post vs 45 minutes post. All other comparisons were significant $(p \leq 0.05$ ).

Rest (between groups) - All comparisons were significant ( $\mathrm{p} \leq 0.05)$.

Rest (experimental) -All comparisons were insignificant $(\mathrm{p}>0.05)$.

2minutes within (between groups) - All comparisons were significant $(\mathrm{p} \leq 0.05)$

2minutes within (experimental) -All comparisons were insignificant $(\mathrm{p}>0.05)$.

4minutes within (between groups) - All comparisons were significant excluding enrollment $(\mathrm{p} \leq 0.05)$. 
4minutes within (experimental) - Enrollment was significantly different than all other values $(\mathrm{p} \leq 0.05)$. All other comparisons were insignificant $(\mathrm{p}>0.05)$.

Immediate-post (between groups) - All comparisons were significant $(\mathrm{p} \leq 0.05)$.

Immediate-post (experimental) - All comparisons were insignificant $(\mathrm{p}>0.05)$.

5minutes post (between groups) -All comparisons were significant $(\mathrm{p} \leq 0.05)$.

5minutes post (experimental) - Sixteen weeks was significantly different from all other values. All other comparisons were insignificant $(\mathrm{p}>0.05)$.

10minutes post (between groups) - All comparisons were significant $(\mathrm{p} \leq 0.05)$.

10minutes post (experimental) - All comparisons were significant $(\mathrm{p} \leq 0.05)$

15minutes post (between groups) - All comparisons were insignificant $(\mathrm{p}>0.05)$.

15 minutes post (experimental) - Comparisons between enrollment and while trained were significant $(\mathrm{p} \leq 0.05)$. Differences while trained were insignificant $(\mathrm{p}>0.05)$.

45minutes post (between groups) - All comparisons were insignificant $(\mathrm{p}>0.05)$.

45minutes post (experimental) - All comparisons were insignificant $(\mathrm{p}>0.05)$. All data regarding rate of perceived exertion are presented in table 8 .

Table 8 rate of perceived exertion before, during, and after the Six Minute Walk Test according to visit and timing of measurement (Mean \pm SD)

\begin{tabular}{|c|c|c|c|c|}
\hline \multirow{2}{*}{$\begin{array}{l}\text { RPEa } \\
\text { (scale) }\end{array}$} & $\begin{array}{l}\text { Enroll } \\
\text { healthy }\end{array}$ & $\begin{array}{l}\text { Enroll } \\
\text { OSA }\end{array}$ & EXS+8Wk & EXS+16Wk \\
\hline & \multicolumn{2}{|c|}{ No intervention } & \multicolumn{2}{|c|}{ Exercise intervention } \\
\hline $\mathbf{N}$ & 25 & 25 & 25 & 25 \\
\hline Rest $\dagger$ & $6.08 \pm 0.27$ & $6.92 \pm 0.57$ & $6.7 \pm 0.5$ & $6.63 \pm 0.22$ \\
\hline$+2 \min$ & $9.28 \pm 0.78$ & $13.5 \pm 1.63$ & $12.08 \pm 2.09$ & $12.1 \pm 1.98$ \\
\hline$+4 \min$ & $13.18 \pm 1.23$ & $14.12 \pm 1.5$ & $12.08 \pm 2.09$ & $12.1 \pm 1.98$ \\
\hline $\begin{array}{l}\text { Immediate- } \\
\text { post }\end{array}$ & $13.79 \pm 1.68$ & $16.96 \pm 0.98$ & $16.46 \pm 2.08$ & $16.3 \pm 1.35$ \\
\hline $5 \mathrm{~min}$ post & $9.24 \pm 0.64$ & $14.16 \pm 0.58$ & $14.26 \pm 0.99$ & $13.94 \pm 1.15$ \\
\hline $10 \mathrm{~min}$ post & $6.59 \pm 0.28$ & $11.08 \pm 0.78$ & $9.16 \pm 0.79$ & $8.64 \pm 0.63$ \\
\hline $15 \mathrm{~min}$ post & $6.07 \pm 0.21$ & $8.76 \pm 0.6$ & $6.87 \pm 0.57$ & $6.69 \pm 0.61$ \\
\hline $45 \mathrm{~min}$ post & $6.0 \pm 0.34$ & $6.95 \pm 0.62$ & $6.66 \pm 0.42$ & $6.7 \mathrm{I} \pm 0.44$ \\
\hline
\end{tabular}

$\mathrm{N}$, number of subjects; RPE, rate of perceived exertion; SD, standard deviation; $\dagger$, values obtained while sitting; a, scale of 6-20; EXS, exercise; Wk, weeks;

\section{Lactate $(\mathrm{mmol} \cdot \mathrm{L}-\mathrm{I})$}

Rest to 45minutes post (control) - Multiple comparisons were insignificant $(p>0.05)$ including rest vs 10 minutes post; rest vs 15 minutes post; rest vs 45 minutes post; 10 minutes post vs 15 minutes post; 10 minutes post vs 15 minutes post; and 15 minutes post vs 45 minutes post. All other comparisons were significant $(\mathrm{p} \leq 0.05)$.
Rest to 45minutes post (experimental; Enrollment) - Multiple comparisons were insignificant ( $\mathrm{p}>0.05$ ) including rest vs 45 minutes post; immediate-post vs 5 minutes post; and 10 minutes post vs 45 minutes post. All other comparisons were significant $(\mathrm{p} \leq 0.05)$.

Rest to 45minutes post (experimental; +8weeks) - Multiple comparisons were insignificant $(\mathrm{p}>0.05)$ including rest vs 15 minutes post; rest vs 45 minutes post; immediate-post vs 5 minutes post; 5 minutes post vs 10 minutes post; and 15 minutes post vs 45 minutes post. All other comparisons were significant $(\mathrm{p} \leq 0.05)$.

Rest to 45minutes post (experimental; +16weeks) - Multiple comparisons were insignificant ( $\mathrm{p}>0.05$ ) including rest vs 15 minutes post; rest vs 45 minutes post; immediate-post vs 5 minutes post; and 15 minutes post vs 45 minutes post. All other comparisons were significant $(\mathrm{p} \leq 0.05)$

Rest (between groups) - All comparisons were insignificant excluding after sixteen weeks $(\mathrm{p}>0.05)$.

Rest (experimental) -All comparisons were insignificant ( $\mathrm{p}>0.05)$.

Immediate-post (between groups) - All comparisons were significant $(\mathrm{p} \leq 0.05)$.

Immediate-post (experimental) - All comparisons were insignificant $(\mathrm{p}>0.05)$

5minutes post (between groups) - All comparisons were significant $(\mathrm{p} \leq 0.05)$.

5minutes post (experimental) - All comparisons were insignificant $(\mathrm{p}>0.05)$.

10minutes post (between groups) - All comparisons were significant $(\mathrm{p} \leq 0.05)$.

10minutes post (experimental)-All comparisons were insignificant $(\mathrm{p}>0.05)$.

15minutes post (between groups) - All comparisons were significant $(\mathrm{p} \leq 0.05)$

15 minutes post (experimental) -All comparisons were insignificant $(\mathrm{p}>0.05)$.

45minutes post (between groups) -All comparisons were insignificant $(\mathrm{p}>0.05)$.

45minutes post (experimental) -All comparisons were insignificant $(\mathrm{p}>0.05)$. All data regarding rate of lactate are presented in Table 9.

Table 9 Lactate before and after the Six Minute Walk Test according to visit and timing of measurement (Mean $\pm S D$ )

\begin{tabular}{|c|c|c|c|c|}
\hline \multirow{2}{*}{$\begin{array}{l}\text { Lactate } \\
\left(\mathrm{mmol} \mathrm{L}^{-1}\right)\end{array}$} & $\begin{array}{l}\text { Enroll } \\
\text { healthy }\end{array}$ & $\begin{array}{l}\text { Enroll } \\
\text { OSA }\end{array}$ & EXS+8Wk & EXS+16Wk \\
\hline & \multicolumn{2}{|c|}{ No intervention } & \multicolumn{2}{|c|}{ Exercise intervention } \\
\hline $\mathbf{N}$ & 25 & 25 & 25 & 25 \\
\hline Rest $†$ & $\mathrm{I} .73 \pm 0.4 \mathrm{I}$ & $2.01 \pm 0.5$ & $1.95 \pm 0.53$ & $1.96 \pm 0.56$ \\
\hline $\begin{array}{l}\text { Immediate- } \\
\text { post }\end{array}$ & $5.15 \pm 0.87$ & $2.93 \pm 0.79$ & $3.03 \pm 0.66$ & $3.21 \pm 0.92$ \\
\hline 5 min post & $3.7 \pm 0.53$ & $2.85 \pm 0.63$ & $2.9 \pm 0.75$ & $2.95 \pm 0.8$ \\
\hline
\end{tabular}


Table continued

\begin{tabular}{|c|c|c|c|c|}
\hline \multirow{2}{*}{ 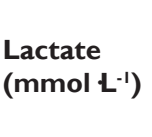 } & $\begin{array}{l}\text { Enroll } \\
\text { healthy }\end{array}$ & $\begin{array}{l}\text { Enroll } \\
\text { OSA }\end{array}$ & EXS+8Wk & EXS+16Wk \\
\hline & \multicolumn{2}{|c|}{ No intervention } & \multicolumn{2}{|c|}{ Exercise intervention } \\
\hline I0min post & $1.89 \pm 0.47$ & $2.44 \pm 0.7$ I & $2.57 \pm 0.58$ & $2.56 \pm 0.66$ \\
\hline $15 \mathrm{~min}$ post & $1.69 \pm 0.32$ & $2.38 \pm 0.4 \mathrm{I}$ & $1.97 \pm 0.46$ & $1.96 \pm 0.33$ \\
\hline $45 \mathrm{~min}$ post & $1.75 \pm 0.45$ & $1.98 \pm 0.37$ & $1.96 \pm 0.39$ & $1.95 \pm 0.4 \mid$ \\
\hline
\end{tabular}

$\mathrm{N}$, number of subjects; mmol, millimole; L, liter; SD, standard deviation; $\dagger$, values obtained while sitting; EXS, exercise; $\mathrm{Wk}$, weeks

\section{Angina (scale)}

No angina events were noted for the control group upon enrollment while five events occurred throughout the study in the experimental group. Number of events for experimental group was significantly higher compared to control group ( 2 vs 0 events $\cdot \operatorname{session}^{-1}$, respectively, $\mathrm{p} \leq 0.05$ ). All angina events throughout the study occurred within four minutes and/or immediate-post; the number of events within four minutes was significantly lower while compared to immediate-post ( 1 vs 4 events; $20 \%$ vs $80 \%$ of events, respectively, $\mathrm{p} \leq 0.05$ ). Angina events were almost completely equally distributed between data collection sessions with an insignificant difference ( 2 vs 1 vs 1 vs 1 events'session ${ }^{-1}$, respectively; $\mathrm{p}>0.05$ ).

\section{Dyspnea (scale)}

No dyspnea events were noted for the control group upon enrollment while ten events occurred throughout the study in the experimental group. Number of events for experimental group was significantly higher compared to control group (10 vs 0 events·study ${ }^{-1}$, respectively, $\mathrm{p} \leq 0.01)$. The most events occurred immediate-post, while an equal number of events occurred at rest and within four minutes ( 6 vs 2 vs 2 events, respectively). The most events occurred upon enrollment, while events decreased as time passed by (4 vs 3 vs 2 vs 1 events session $^{-1}$, respectively). Number of events were significantly higher while untrained compared to while trained ( 7 vs 3 events'session ${ }^{-1}$, respectively; $\mathrm{p} \leq 0.05$ ). Within the untrained and trained weeks, no significant differences were noted ( $p>0.05)$. While comparing the number of events according to the timing within the sessions, only one comparisons was insignificant. The number of events at rest was the same as that within four minutes ( 2 vs 2 events, respectively; $p>0.05$ ). All other comparisons were significant $(\mathrm{p} \leq 0.05)$.

\section{Angina vs Dyspnea}

Total number of events throughout the study was significantly higher for dyspnea compared to angina (10 vs 5 events'study ${ }^{-1}$, respectively; $\mathrm{p} \leq 0.05$ ). Total number of events upon enrollment was insignificantly higher for dyspnea compared to angina (4 vs 2 events'session ${ }^{-1}$, respectively; $\mathrm{p}>0.05$ ). Total number of events at sixteen weeks was insignificantly higher for dyspnea compared to angina (3 vs 1 events'session ${ }^{-1}$, respectively; $\mathrm{p}>0.05$ ). Total number of events after eight weeks of exercise was insignificantly higher for dyspnea compared to angina (2 vs 1 events'session ${ }^{-1}$, respectively; $\mathrm{p}>0.05$ ). Total number of events after sixteen weeks of exercise was identical for dyspnea and angina ( 1 vs 1 events session $^{-1}$, respectively; $\mathrm{p}>0.05$ ). All data regarding rate of angina and dyspnea events are presented in Table 10

Table 10 number of Angina and dyspnea eventsa, b before, during, and after the Six Minute Walk Test according to visit and timing of measurement (Mean \pm SD)

\begin{tabular}{|c|c|c|c|c|c|c|c|c|c|c|}
\hline \multirow{3}{*}{$\begin{array}{l}\text { Timing of events } \\
\mathrm{N}\end{array}$} & \multicolumn{2}{|c|}{ Enroll healthy } & \multicolumn{2}{|c|}{ Enroll OSA } & \multicolumn{2}{|c|}{ EXS+8 Wk } & \multicolumn{2}{|c|}{$\begin{array}{l}\text { EXS+16 } \\
\text { Wk }\end{array}$} & \multirow{4}{*}{ Sum Ang } & \multirow{4}{*}{ Sum Dys } \\
\hline & \multicolumn{4}{|c|}{ No intervention } & \multicolumn{4}{|c|}{ Exercise intervention } & & \\
\hline & 25 & & 25 & & 25 & & 25 & & & \\
\hline Events $^{\dagger}$ & Ang & Dys & Ang & Dys & Ang & Dys & Ang & Dys & & \\
\hline $\operatorname{Rest}^{\dagger}$ & 0 & 0 & 0 & I & 0 & 0 & 0 & 0 & 0 & 2 \\
\hline$+2 \min$ & 0 & 0 & 0 & 0 & 0 & 0 & 0 & 0 & 0 & 0 \\
\hline$+4 \min$ & 0 & 0 & I & I & 0 & 1 & 0 & 0 & $\mathbf{I}$ & 2 \\
\hline Immediate-post & 0 & 0 & I & 2 & 1 & 1 & 1 & I & 4 & 6 \\
\hline $5 \mathrm{~min}$ post & 0 & 0 & 0 & 0 & 0 & 0 & 0 & 0 & 0 & 0 \\
\hline 10 min post & 0 & 0 & 0 & 0 & 0 & 0 & 0 & 0 & 0 & 0 \\
\hline $15 \mathrm{~min}$ post & 0 & 0 & 0 & 0 & 0 & 0 & 0 & 0 & 0 & 0 \\
\hline $45 \mathrm{~min}$ post & 0 & 0 & 0 & 0 & 0 & 0 & 0 & 0 & 0 & 0 \\
\hline Sum & 0 & 0 & 2 & 4 & I & 2 & I & I & 5 & 10 \\
\hline
\end{tabular}

$\mathrm{N}$, number of subjects; SD, standard deviation; $\dagger$, values obtained while sitting; a, grading of 3 at least on the Angina scale; $b$, grading of 4 on the modified Borg dyspnea scale; Ang, Angina; Dys, dyspnea; $\dagger$, in absolute cases; EXS, exercise; Wk, weeks; 


\section{The 6MWT}

6MWT distance $(\mathrm{m} ; \mathrm{Ft})$ : The control group achieved a significantly greater distance compared to the experimental group's distances in all sessions $(\mathrm{p} \leq 0.05)$. A significant difference was noted for the distance achieved within the experimental group after sixteen weeks of exercise compared to all other sessions $(\mathrm{p} \leq 0.05)$. All other comparisons within the experimental group were insignificant $(\mathrm{p}>0.05)$.

Walking speed (kph; mph): The control group achieved a significantly greater walking speed compared to that achieved by the experimental group in all sessions $(\mathrm{p} \leq 0.05)$. A significant difference was noted for the walking speed achieved within the experimental group after sixteen weeks of exercise compared to all other sessions $(p \leq 0.05)$. All other comparisons within the experimental group were insignificant $(\mathrm{p}>0.05)$.

Test terminations (number): No tests were terminated at all upon enrollment of the control group. Thus, all comparisons between groups for the number of terminations per study were significant (15 vs 0 terminations $\cdot$ study $^{-1} ; \mathrm{p} \leq 0.05$ ). The number of tests terminated by the research team was significantly greater compared to those by the subjects ( 13 vs 2 terminations $\cdot$ study $^{-1} ; \mathrm{p} \leq 0.05$ ). The number of tests terminated by the research team was significantly greater while subjects were untrained compared to while trained (10 vs 3 terminations $\cdot$ study $^{-1}$, respectively, $\mathrm{p} \leq 0.05$ ). The number of terminations by the researchers was insignificantly lower after eight weeks of exercise compared to after sixteen weeks of exercise (1 vs 2 terminations'session ${ }^{-1}$, respectively, $\mathrm{p}>0.05$ ). The number of terminations by the researchers upon enrollment was significantly higher in comparison to both sessions while exercised (6 vs 1 and 6 vs 2 , terminations session $^{-1}$, respectively; $p \leq 0.05$ ). The number of terminations by the researchers after sixteen weeks was insignificantly higher in comparison to both sessions while exercised ( 4 vs 1 and 4 vs 2 terminations $\cdot \operatorname{session}^{-1}$, respectively; $p>0.05$ ). All other comparisons were insignificant $(\mathrm{p}>0.05)$. No significant difference in the number of terminations per session by subjects was found ( 0 vs 0 vs 2 vs 0 terminations $\cdot$ session $\left.^{-1} ; p>0.05\right)$. The number of terminations between researchers and subjects upon enrollment was significantly greater for the researchers (6 vs 0 terminations $\cdot \operatorname{session}^{-1}$, respectively; $\mathrm{p} \leq 0.05$ ). The number of terminations between researchers and subjects after sixteen weeks was significantly greater for the researchers (4 vs 0 terminations session $^{-1}$, respectively; $\mathrm{p} \leq 0.05$ ). The number of terminations between researchers and subjects after eight weeks of exercise was insignificantly lower for the researchers ( 1 vs 2 terminations $\cdot \operatorname{session}^{-1}$, respectively; $p>0.05$ ). The number of terminations between researchers and subjects after sixteen weeks of exercise was insignificantly higher for the researchers ( 2 vs 0 terminations $\cdot \operatorname{session}^{-1}$, respectively; $p>0.05$ ).

Sitting down (number): The average amount of times subjects sat down during the test was significantly lower for the control group compared to the experimental group $(\mathrm{p} \leq 0.01)$ since none of the control subjects sat down during the test. All other comparisons were found to be significant $(\mathrm{p} \leq 0.05)$.

Duration of sitting down (seconds): The average duration of sitting down during the test was significantly lower for the control group compared to the experimental group $(\mathrm{p} \leq 0.01)$ since none of the control subjects sat down during the test. The duration of sitting down during the test (for each time a subject sat down) was significantly higher while untrained compared to while trained within the experimental group. Differences in sitting down duration while untrained and while trained were insignificant $(\mathrm{p}>0.05)$. All data regarding indices of the 6MWT are presented in table 11

Table II Six Minute Walk Test related variables according to visit and timing of measurement (Mean \pm SD)

\begin{tabular}{|c|c|c|c|c|}
\hline \multirow{2}{*}{ Distance } & Enroll healthy & Enroll OSA & EXS+8Wk & EXS+16Wk \\
\hline & \multicolumn{2}{|l|}{ No intervention } & \multicolumn{2}{|c|}{ Exercise intervention } \\
\hline $\mathbf{N}$ & 25 & 25 & 25 & 25 \\
\hline Distance $(\mathrm{m})$ & $579.6 \pm 98.41 \mathrm{p}$ & $319.53 \pm 44.13 p$ & $345.6 \pm 82.4 p$ & $388.79 \pm 75.5 p$ \\
\hline Distance $(\mathrm{Ft})^{\mathrm{a}}$ & $1901.57 \pm 322.86 p$ & $1048.32 \pm 144.78 p$ & $1133.85 \pm 270.34 p$ & $1275.55 \pm 247.7 p$ \\
\hline Improvement $(\mathrm{m})^{\ddagger}$ & $\mathrm{N} / \mathrm{A}$ & $\mathrm{N} / \mathrm{A}$ & $26.07 \pm 63.7 n$ & $69.26 \pm 58.42 p$ \\
\hline Improvement $(\mathrm{Ft})^{\ddagger}$ & $\mathrm{N} / \mathrm{A}$ & N/A & $85.53 \pm 208.6 n$ & $227.23 \pm 192.2 p$ \\
\hline Walking speed (kmph) & $5.8 \pm 0.98$ & $3.2 \pm 0.44$ & $3.45 \pm 0.82$ & $3.88 \pm 0.75$ \\
\hline Walking speed (mph) & $3.6 \pm 0.61$ & $1.99 \pm 0.27$ & $2.15 \pm 0.5 \mid$ & $2.42 \pm 0.47$ \\
\hline $\begin{array}{l}\text { Number of test terminated by research } \\
\text { team; \% }\end{array}$ & $0^{\dagger}$ & $6(100 \%)^{\dagger}$ & I $(33 \%)^{\dagger}$ & $2(100 \%)^{\dagger}$ \\
\hline Number of test terminated by subjects; $\%$ & $0^{\dagger}$ & $0(0 \%)^{\dagger}$ & $2(67 \%)^{\dagger}$ & $0(0 \%)^{\dagger}$ \\
\hline $\begin{array}{l}\text { Number of times subjects sat down } \\
\text { during the test } \mathrm{t}^{\mathrm{b}}\end{array}$ & $0^{\dagger}$ & $0.98 \pm 0.23$ & $0.82 \pm 0.07$ & $0.78 \pm 0.3$ \\
\hline $\begin{array}{l}\text { Duration of sitting down during the test } \\
\text { (seconds) }\end{array}$ & $0^{\dagger}$ & $31.6 \pm 5.78$ & $22.39 \pm 3$ & $24.0 \pm 3.4$ \\
\hline
\end{tabular}




\begin{tabular}{|c|c|c|c|c|}
\hline \multirow{2}{*}{ Distance } & Enroll healthy & Enroll OSA & EXS+8Wk & EXS+I6Wk \\
\hline & \multicolumn{2}{|l|}{ No intervention } & \multicolumn{2}{|c|}{ Exercise intervention } \\
\hline $\mathbf{N}$ & 25 & 25 & 25 & 25 \\
\hline Walking distance lost $\left(\mathrm{m} \cdot\right.$ test $\left.^{-1}\right)$ & 0 & 8.36 & 4.95 & 5.05 \\
\hline Walking distance lost $\left(\mathrm{ft} \cdot\right.$ test $^{-1}$ ) & 0 & 27.42 & 16.24 & 16.56 \\
\hline
\end{tabular}

$\mathrm{N}$, number of subjects; SD, standard deviation; a, calculated as the distance in meters divided by 30.48 ; b, average per subject, per test; c, average per subject, per occurrence; $\mathrm{p}$, positive prognosis; $\mathrm{n}$, negative prognosis; $\mathrm{m}$, meters; Ft, feet; $\dagger$, absolute number of occurrence; $\neq$, improvement from distance achieved at enrollment; N/A, non-applicable; kmph, kilometer per hour; mph, miles per hour; EXS, exercise;Wk, weeks;

\section{Spirometry}

FEV1 predicted (\%): FEV1 predicted at rest was significantly higher in the control group compared to all measurements of the experimental group ( $\mathrm{p} \leq 0.01$ ). A significant difference was noted for the comparison of values at rest between sixteen weeks of exercising vs enrollment within the experimental group $(\mathrm{p} \leq 0.05)$. All other comparisons at rest were insignificant $(\mathrm{p}>0.05)$ table 12.

Table 12 spirometry related variables according to visit (Mean \pm SD)

\begin{tabular}{lllll}
\hline \multirow{2}{*}{ Variable } & $\begin{array}{l}\text { Enroll } \\
\text { healthy }\end{array}$ & $\begin{array}{l}\text { Enroll } \\
\text { OSA }\end{array}$ & $\begin{array}{l}\text { EXS+8 } \\
\text { Wk }\end{array}$ & $\begin{array}{l}\text { EXS+16 } \\
\text { Wk }\end{array}$ \\
\cline { 2 - 5 } & \multicolumn{2}{l}{ No intervention } & \multicolumn{2}{l}{ Exercise intervention } \\
\cline { 2 - 5 } N & 25 & 25 & 25 & 25 \\
\hline
\end{tabular}

\section{FEVI}

predictedR

$101.96 \pm 4.3$

$63.42 \pm 4.51$

$67.5 \pm 5.23$

$69.88 \pm 4.49$

(\%)

\section{FEVI}

\begin{tabular}{lcccc}
$\begin{array}{l}\text { predictedIP } \\
(\%)^{\dagger}\end{array}$ & $97.3 \pm 2.7$ & $75.3 \pm 3.3$ & $76.37 \pm 5.16$ & $79.11 \pm 4.18$ \\
$\begin{array}{l}\text { FVC } \\
\begin{array}{l}\text { predictedR } \\
(\%)^{\dagger}\end{array}\end{array}$ & $102.63 \pm 4.78$ & $68.23 \pm 4.09$ & $71.03 \pm 3.79$ & $74.63 \pm 3.61$ \\
$\begin{array}{l}\text { FVC } \\
\text { predictedIP } \\
\text { (\%) }\end{array}$ & $98.23 \pm 1.68$ & $76.44 \pm 6.5$ & $80.67 \pm 4.35$ & $84.22 \pm 3.67$ \\
$\begin{array}{l}\text { FEVI/FVCR } \\
\text { ratio }\end{array}$ & $0.753 \pm 0.78$ & $0.657 \pm 0.9$ & $0.693 \pm 0.71$ & $0.719 \pm 0.8$ \\
$\begin{array}{l}\text { FEVI/FVCIP } \\
\text { ratio }\end{array}$ & $0.84 \pm 0.54$ & $0.79 \pm 1.5$ & $0.81 \pm 1.97$ & $0.82 \pm 3.51$ \\
\hline
\end{tabular}

$\mathrm{N}$, number of subjects; SD, standard deviation; FEV, forced expiratory volume; FVC, forced vital capacity; R, at rest; IP, immediate-post; EXS, exercise; Wk, weeks; $\dagger$, compared for the predicted value of an age and sex matching healthy person

FEV1 predicted immediate-post was significantly higher in the control group compared to all measurements of the experimental group ( $\mathrm{p} \leq 0.05)$. A significant difference was noted for the comparison of values immediate-post between sixteen weeks of exercising vs sixteen weeks within the experimental group $(\mathrm{p} \leq 0.05)$. All other comparisons at rest were insignificant $(p>0.05)$. In the transition from rest to immediate-post, FEV1 predicted was insignificantly higher at rest compared to immediate-post within the control group ( $p>0.05)$.

All comparisons within the experimental group were significant while comparing values at rest with values immediate-post $(\mathrm{p} \leq 0.05)$, whereas values at rest were lower than immediate-post.

FVC predicted (\%): FVC predicted at rest was significantly higher in the control group compared to all measurements of the experimental group $(p \leq 0.05)$. A significant difference was noted for the comparison of values at rest between sixteen weeks of exercising vs enrollment within the experimental group $(\mathrm{p} \leq 0.05)$. All other comparisons at rest were insignificant $(p>0.05)$.

FVC predicted immediate-post was significantly higher in the control group compared to all measurements of the experimental group $(\mathrm{p} \leq 0.05)$. A significant difference was noted for the comparison of values immediate-post between sixteen weeks of exercising vs sixteen weeks within the experimental group $(p \leq 0.05)$. All other comparisons at rest were insignificant ( $p>0.05$ ). In the transition from rest to immediate-post, FVC predicted was insignificantly higher at rest compared to immediate-post within the control group ( $p>0.05)$.

All comparisons within the experimental group were significant while comparing values at rest with values immediate-post $(p \leq 0.05)$, whereas values at rest were lower than immediate-post.

\section{FEVI/FVC ratio}

FEV1/FVC ratio at rest was significantly higher in the control group compared to all measurements of the experimental group $(p \leq 0.05)$ excluding after sixteen weeks of exercise $(p>0.05)$. Significant differences were noted for the comparisons of values at rest between sixteen weeks of exercising vs enrollment and sixteen weeks $(\mathrm{p} \leq 0.05)$, and an insignificant difference compared to eight weeks of exercise $(p>0.05)$ within the experimental group. All other comparisons at rest were insignificant $(p>0.05)$.

FEV1/FVC ratio immediate-post was significantly higher in the control group compared ratio upon enrollment and sixteen weeks of the experimental group $(\mathrm{p} \leq 0.05)$. A significant difference was noted for the comparison of values immediate-post between sixteen weeks of exercising vs enrollment within the experimental group $(\mathrm{p} \leq 0.05)$. All other comparisons at rest were insignificant $(\mathrm{p}>0.05)$. In the transition from rest to immediate-post, FEV1/FVC ratio was significantly higher immediate-post compared to rest within the control group ( $p>0.05)$. All comparisons within the experimental group were significant while comparing values at rest with values immediate-post $(\mathrm{p} \leq 0.05)$, 
whereas values at rest were lower than immediate-post.

\section{Discussion}

\section{In general}

The influence of prolonged aerobic exercise on s-klotho levels in people with severe obstructive sleep apnea has never been investigated prior to this study, especially in such length. The present study demonstrates clear and significant influences of sixteen weeks of aerobic training on s-klotho, hemodynamic responses, and spirometry indices before, during, and after the Six Minute Walk Test.

Experimental group's BMI was significantly greater compared to age and sex matching healthy subjects. Obesity $\left(\mathrm{BMI}>30 \mathrm{~kg} \cdot \mathrm{m}^{-2}\right)$ has been previously indicated in countless publications as a risk factor and predictor of future and current sleep apnea. ${ }^{33-38}$

An additional risk factor well established in the literature is a person's neck circumference. Previous data published indicated a neck circumference of sixteen inches or higher as a possible risk factor for obstructive sleep apnea. Furthermore, the neck circumference to height ratio is indicated as better predictor than BMI alone. ${ }^{37-39}$ As with the OSA subjects of this study, previous studies have shown little to no difference in Weight and BMI following exercise and/or stretching. ${ }^{40,41}$ While the design of the study did not allow full control of diet and adherence to exercise, the lack of improvement in BMI and neck circumference may be explained by possible lack of adherence to instructions throughout the study. Exercise alone has been shown as unable to battle bad nutritional habits. ${ }^{42,43}$

In this current study, exercise had a positive effect on AHI decreasing it by five episodes/hour in average. This data is perfectly aligned with the literature, indicating improvements in AHI of up to 6 episodes/hour. ${ }^{44,45}$

\section{S-klotho}

To the best of the researchers' knowledge, no previously published data exist regarding OSA, s-klotho, and/or the effects of exercise, nor the combination of. The data of this study suggest that s-klotho is significantly higher in healthy age and sex matching subject before and after exercise compared to subjects with OSA. Similar findings between healthy and clinical populations have been noted. ${ }^{46-48}$

While resistance training showed no to little effect on s-klotho levels, aerobic training, especially chronic aerobic training has been proven to increase the biomarker's levels. ${ }^{25,46-48}$ The current study further suggest that s-klotho levels increase from baseline to immediate-post exercise and then decrease. Levels of s-klotho are higher immediatepost and decrease faster in healthy compared to OSA subjects. No published data exists to compare with in current literature. It is the researchers' suggestion that biomarker's levels remain high in clinical populations in order to better battle high levels of ROSs induced by exercise.

\section{Heart rate}

Heart rate was higher in the OSA subjects compared to that of the control group. These data are in accordance with the known heart rate for the population. ${ }^{49}$ During the 6MWT, healthy subjects were able to move faster and reach a higher heart rate on comparison to subjects with OSA. Furthermore, the decline in heart rate during recovery was slower in subjects with OSA. Such dynamics have been previously reported in regard to comparison of healthy vs clinical, and specifically in regard to people with OSA..$^{50-55}$ Sixteen weeks of exercise improved heart at rest and responses during exercise in the subjects with OSA. Such improvement to heart rate were previously noted in the literature. ${ }^{53,56}$ As in previous studies, the data of this study suggest a blunt response to exercise and during exercise, preventing people with OSA to function at levels common to age and sex matching healthy people..$^{53}$

\section{Systolic blood pressure}

Systolic blood pressure at rest was lower in the control group compared to people with OSA, and has improved in the experimental group as a response to exercise. Such results have been previously reported in regards to people with OSA and exercise. ${ }^{53,57,58}$

Systolic blood pressure seemed to be somewhat lower than previously reported blood pressure values in men with OSA, yet lower than hypertensive men with OSA. ${ }^{53,57,59}$ As seen in heart rate's response immediate-post, so was a blunt response seen during exercise and immediate-post exercise regarding systolic blood pressure. ${ }^{53-55,57,59}$

\section{Diastolic blood pressure}

Diastolic blood pressure at rest was higher for subjects with OSA ompared to controls, yet within the range of values previously reported for age and sex matching people..$^{53,54,57-60}$ Diastolic blood pressure within the experimental group decreased due to exercise. Such a decreased has been previously noted in similar subjects with OSA. ${ }^{55,58,60,62}$ Both control and experimental groups' diastolic blood pressure was within resting values within ten minutes post. The reported values during recovery seem to be similar to previously reported. ${ }^{62}$ The researchers suggest that the same impairments mentioned and referenced earlier, cause diastolic blood pressure to return to the vicinity of resting values within ten minutes post.

\section{Oxygen saturation}

Oxygen saturation was lower in people with OSA compared to the control subjects. Multiple studies have shown oxygen saturation to be lower in people with OSA at rest. ${ }^{63-65}$

Oxygen saturation has improved following the sixteen weeks of exercise. These findings are documented in the literature regarding adaptation to exercise in people with OSA. ${ }^{66,67}$ During exercise, oxygen saturation in the OSA subjects declined, and rose gradually post-exercise. Such dynamics have been previously reported. ${ }^{63-67}$

\section{Rate of perceived exertion}

RPE was greater in subjects with OSA compared to the control group from rest to recovery measurements and during all data collection sessions. While the researchers were unable to find data equivalent in it extent to that of this study in subjects undergoing the 6MWT, it has been suggested that the perception of dyspnea is increased during and after exercise in people with OSA. ${ }^{51}$ Since such a perception is one of perceived stress, it is possible that in people with OSA, nonetheless severe OSA, the perception of dyspnea may be influencing their perception of their rate of exertion. Inconsistency in RPE has been noted in healthy people ${ }^{68}$ and in people with COPD, often compared to people with OSA, since people with COPD may be diagnosed as OSA as well, and vice versa, as well as the obstructive aspects. ${ }^{69-71}$ 


\section{Angina}

Angina events during this study were based upon the subjects' rating utilizing the angina scale. Angina events in people with OSA are not surprising. Evidence exists suggesting that people with OSA may perceive themselves as experiencing an angina event, similar to that of cardiac patients. ${ }^{72}$ On the other hand, OSA has been associated with CAD ${ }^{73,74}$ Furthermore, OSA has been suggested as a risk marker for CAD. ${ }^{75}$ Thus, while few angina events occurred throughout the study, the researchers suggest the "occurrence" of angina events during the study were either real or perceived as real, while such events could have been related to undiagnosed CAD or other cardiac clinical conditions. ${ }^{76}$ The angina events were concentrated within four minutes of the start of the 6MWT and/or immediate-post. Angina events occurred in 5\% (5/100 test) of the 6MWT within the experimental group. A similar percentage of events was previously reported in a large cohort $(n=740)$ of people with chronic lung disease. ${ }^{77}$ The number of angina events decreased while trained compared to while untrained. The ability of exercise to reduce the occurrence of angina in clinical populations has been previously noted..$^{78,79}$

\section{Dyspnea}

Ten dyspnea events occurred (or were perceived as occurring) throughout this study. The dyspnea events were concentrated within four minutes of the start of the 6MWT and/or immediate-post. Dyspnea events occurred in 10\% (10/100 test) of the 6MWT within the experimental group. In previous studies one-third of the subjects reported experiencing dyspnea during the 6MWT while others did not. ${ }^{80,81}$ With that said, the occurrence of dyspnea during exercise in people with OSA is not at all a new finding. ${ }^{51,80,82}$ The highest number of events occurred while untrained and immediate-post. Dyspnea upon maximal effort has been previously noted in several studies pertaining to people with OSA. ${ }^{83,84}$ Yet, exercise has been indicated as one of several means to reduce dyspnea events in people with obstructive diseases. ${ }^{55,85-87}$ These previous findings are a basis for understanding the occurrence of dyspnea in this study and the improvement following weeks of exercise.

\section{Distance achieved in the 6MWT}

The distance achieved by both groups of this study were within previously reported ranges for healthy vs people with OSA. ${ }^{51,88}$ Furthermore, both the fact that healthy people achieve better distance compared to people with clinical conditions, and that exercise improves the distance achieved during the 6MWT have been reposted before. ${ }^{51,88-90}$ The effect of exercise on the distance achieved during the 6MWT has also been noted in people with OSA..$^{91-93}$ Indeed, the results of this current study show clearly that the control groups' distance achieved during the 6MWT was greater than that of the subjects with OSA, and that the distance has improved after exercise within the experimental group.

Subject with OSA have improved their achieved distance by 2.5 meters in average while untrained. This improvement, though not substantial, can be the result of better strategy and/or learning from one test to the other, and perhaps even random. Rather or not the prognosis becomes positive or negative has been shown to be dependent on the overall distance achieved during the test, whereas a distance $>300$ meters or more yields a positive prognosis and $<300$ yields a negative prognosis. ${ }^{94-96}$ The improvement (meters) between two tests of the same person has been reported to have prognosis value in several different clinical populations. ${ }^{97,98}$ The minimal improvement in the distance, also termed the "minimal clinical important difference (MCID) achieved during the 6MWT in order for the prognosis to be positive is different for different clinical populations yet ranges from 30meters to 80 meters between tests of the same person. ${ }^{94-99}$ The results of the current study show that while sixteen weeks of aerobic training achieved the minimum improvement needed for a positive prognosis, eight weeks were not a strong enough stimulus, though subjects achieved $>300$ meters distance in all tests.

\section{Walking speed during the 6MWT}

Since the duration of movement is fixated during the test to a maximum of 6minutes, and the distance achieved is known as part of the results of this study, mathematically, the average speed is already determined. Thus, the analysis of walking speed between groups and within the experimental group does not differ than that of the distance achieved, nor the minimal distance needed to be achieved to result in a positive prognosis. It has been suggested that walking speed may be affected and regulated according to will to avoid dyspnea, leading to a lower walking speed. ${ }^{100}$

\section{Sitting down duration}

While the phenomenon of sitting down during the 6MWT is well known and is addressed in the guidelines for the administration of the test, the researchers were unable to find data pertaining to the average duration of each sitting down episode. The time spent sitting down (average per single sitting down episode) ranged in this study from $\approx 22$ to $\approx 33$ seconds. These durations translate to a loss of walking distance of $\approx 4.95$ to $\approx 9.82\left(\mathrm{~m} \cdot\right.$ test $\left.^{-1}\right)$. The decrease in distance loss with the onset of training is an additional indication of the positive influences of exercise on functional aspects of people with severe OSA as established previously in this discussion.

\section{FEVI predicted (\%)}

Often, obstructive pulmonary diseases are accompanied by sere OSA, and resemble in functional indices and responses to exercise. ${ }^{101,102}$ The extent of data pertaining to people with COPD is substantially greater in comparison to that of OSA. Furthermore, the need to define OSA as a disease within itself and a treatment plan of its own has been discussed previously. ${ }^{103,104}$ It is for these reasons that the two are often regarded together or as clinical conditions with similar symptoms, as well as risk factor for each other, known as the "overlap syndrome". ${ }^{101,102}$ The control group had a higher FEV1 predicted compared to that of the experimental group. Suspectedhealthy having a higher FEV1 predicted has been noted in the professional literature before. ${ }^{105-107}$ Sixteen weeks of aerobic exercise elevated FEV1 predicted by roughly $6 \%$ at rest. The positive effects of exercise on spirometry indices have been reported before whereas adaptation to exercise induced an increase in FEV1 predicted (\%). ${ }^{108}$ FEV1 predicted (\%) was elevated from rest to immediate-post in the experimental group yet was decreased in the control group. Such finding have been reported in people with COPD. ${ }^{109}$

\section{FVC predicted (\%)}

The fact that FVC predicted (\%) was higher in the control group at rest compared to that of the experimental group for all sessions is well in accordance with the literature. ${ }^{110,111}$ This variable has also been associated with the distance achieved during the 6MWT whereas shorter distances achieved were highly correlated with lower FVC 
predicted $(\%){ }^{88}$ The same dynamic was observed in the current study. The results of this study indicate that while the control groups $\mathrm{FVC}$ predicted (\%) values were in the range reported for sex and age matching people, that of the experimental group were significantly lower. ${ }^{12,113}$ FVC predicted (\%) improved due to exercise and was increased by $\approx 6 \%$ at rest. Such improvement are well established in the literature. ${ }^{114-116}$ Value decreased mildly in the healthy subjects while increased in the experimental group's subjects in the transition from rest to immediate-post. Several studies have indicated similar dynamics while comparing healthy and clinical populations. ${ }^{117-119}$

\section{FEVI/FVC ratio}

The results of the current study indicate a higher ratio in the control group compared to the experimental group at rest. These results align with previous finding in the literature. ${ }^{120}$ Furthermore, the subjects with OSA had a lower ratio at rest and immediate-post exercise while compared to the control group. In both cases, the experimental group's values improved in response to exercise. Previous studies have shown similar dynamics. ${ }^{121,122}$ A study from 2015 suggests that there are no differences between obese and obese people with OSA regarding their ratio. A possible conclusion could be that obesity within itself is a strong enough reducer of the ratio, regardless of the existence of OSA. ${ }^{11,123}$ Based on this discovery alone and the fact that the OSA subjects of this study were obese, one could anticipate a reduced ratio, actually seen. ${ }^{11,123}$

\section{Conclusions}

Severe OSA has similar symptoms, functional capacities, and responses to exercise as people with COPD have. As shown in previous studies, people with severe OSA respond to exercise by improving vital indices of function.

During the study, subject with severe OSA performed at a lower level compared to age and sex matching subjects, presenting with impaired function during and after the 6MWT. As a result, s-klotho were substantially lower at rest, during exercise, and after adaptation to exercise, while compared to suspected healthy age and sex matching people. As seen in other clinical populations, Aerobic exercise is a powerful adaptation inducer, leading to improvement in multiple parameters, including s-klotho. The researchers wish to call upon others to obtaine more data pertaining to the influence of exercise on people with OSA in general, and in more detail throughout exercise testing.

\section{Acknowledgements}

None.

\section{Conflict of interest}

The author declares no conflict of interest.

\section{References}

1. Patrick Y, Alice Lee, Oishik Raha et al. Effects of Sleep Deprivation on Cognitive and Physical Performance in University Students. Sleep Biol Rhythms. 2017;15(3):217-225.

2. Alhola P, Polo-Kantola P. Sleep Deprivation: Impact on Cognitive Performance. Neuropsychiatr Dis Treat. 2007;3(5):553-567.
3. Myles WS. Sleep Deprivation, Physical Fatigue, and the Perception of Exercise Intensity. Med Sci Sports Exerc. 1985;17(5):580-584.

4. Mejri MA, Yousfi N, Hammouda O, et al. One Night of Partial Sleep Deprivation Increased Biomarkers of Muscle and Cardiac Injuries During Acute Intermittent Exercise. J Sports Med Phys Fitness. 2017;57(5):643-651.

5. Benedict C, Vogel H, Jonas W, et al. Gut Microbiota and Glucometabolic Alterations in Response to Recurrent Partial Sleep Deprivation in Normal-Weight Young Individuals. Mol Metab. 2016;5(12):11751186 .

6. Mejri MA, Yousfi N, Mhenni T et al. Does One Night of Partial Sleep Deprivation Affect the Evening Performance During Intermittent Exercise in Taekwondo Players? J Exerc Rehabil. 2016;12(1):47-53.

7. Rångtell FH, Karamchedu S, Andersson P et al. A Single Night of Sleep Loss Impairs Objective but not Subjective Working Memory Performance in a Sex-Dependent Manner. J Sleep Res. 2018;12651.

8. Goldstein-Piekarski AN, Greer SM, Saletin JM et al. Sex, Sleep Deprivation, and the Anxious Brain. J Cogn Neurosci. 2018;30(4):565-578.

9. Eichhorn N. et al. The Role of Sex in Sleep Deprivation Related Changes of Nociception and Conditioned Pain Modulation. Neuroscience. 2017;pii: S0306-4522(17)30697-30698.

10. Jiang J, Zhongyuan Gan, Yuan Li, et al. REM Sleep Deprivation Induces Endothelial Dysfunction and Hypertension in Middle-Aged Rats: Roles of the eNOS/NO/cGMP Pathway and Supplementation with LArginine. PLOS One. 2017;15:12(8).

11. Khan MS, Aouad R, et al. The Effects of Insomnia and Sleep Loss on Cardiovascular Disease. Sleep Med Clin. 2017;12(2):167-177.

12. Thomas SJ, Calhoun D. Sleep, Insomnia, and Hypertension: Current Findings and Future Directions. J Am Soc Hypertens. 2017;11(2):122129.

13. Kamperis K, Hagstroem S, Radvanska E, et al. Excess Diuresis and Natriuresis during Acute Sleep Deprivation in Healthy Adults. Am J Physiol Renal Physiol. 2010;299(2):F404-F411.

14. Rajizadeh MA, Esmaeilpour K, Masoumi-Ardakani Y, et al. Voluntary Exercise Impact on Cognitive Impairments in Sleep-Deprived Intact Female Rats. Physiol Behav. 2018;188:58-66.

15. Zagaar M, Dao A, Alhaider I et al. Corrigendum to Regular Treadmill Exercise Prevents Sleep Deprivation-Induced Disruption of Synaptic Plasticity and Associated Signaling Cascade in the Dentate Gyrus. Mol Cell Neurosci. 2017;88:53.

16. De-Souza JFT, Dáttilo M, De Mello MT. et al. High-Intensity Interval Training Attenuates Insulin Resistance Induced by Sleep Deprivation in Healthy Males. Front Physiol. 2017;8:992.

17. Slutsky AB, Diekfuss JA, Janssen JA et al. The Effects of Low-Intensity Cycling on Cognitive Performance Following Sleep Deprivation. Physiol Behav. 2017;180:25-30.

18. Matsumura $\mathrm{Y}$, Aizawa $\mathrm{H}$, Shiraki-Iida $\mathrm{T}$, et al. Identification of the Human Klotho Gene and its Two Transcripts Encoding Membrane and Secreted Klotho Protein. Biochem Biophys Res Commun.1998;242(3):626-630.

19. Kuro-o M, Matsumura $\mathrm{Y}$, Aizawa H, et al. Mutation of the Mouse Klotho Gene Leads to a Syndrome Resembling Ageing. Nature. 1997;390(6655):45-51.

20. Arking DE, Krebsova A, Macek M Sr, et al. Association of Human Aging with a Functional Variant of Klotho. Proc Natl Acad Sci U S A. 
2002;99(2):856-61.

21. Rodriguez T. Identifying Significant Biological Markers in Klotho Gene Variants Across Wide Ranging Taxonomy. Journal of Molecular Biology Research. 2015;5(1):p11.

22. Xiao NM, Zhang YM, Zheng Q et al. Klotho is a Serum Factor Related to Human Aging. Chin Med J (Engl). 2004;117(5):742-747.

23. Huang CL. Regulation of Ion Channels by Secreted Klotho: Mechanisms and Implications. Kidney Int. 2010;77(10):855-60.

24. Quintero-Platt G, González-Reimers E , Rodríguez-Gaspar M, et al Alpha Klotho and Fibroblast Growth Factor-23 Among Alcoholics. Alcohol Alcohol. 2017;52(5):542-549.

25. Saghiv M. et al. The Impact of 12 Weeks Exercise Training on Circulating Soluble-Klotho and Pro - BNP in Coronary Artery Disease Patients. J Cardiol Vasc Res. 2017;1(1);1-4.

26. Chung CP, Chang YC, Ding Y, et al. $\alpha-$ Klotho Expression Determines Nitric Oxide Synthesis in Response to FGF-23 in Human Aortic Endothelial Cells. PLoS One. 2017;12(5):e0176817.

27. Kuro-o M. Klotho and Aging. Biochimica et Biophysica Acta 2009;1790(10):1049-58.

28. Zelniker TA, Huscher D, Vonk-Noordegraaf A, et al. The 6MWT as a Prognostic Tool in Pulmonary Arterial Hypertension: Results from the COMPERA Registry. Clin Res Cardiol. 2018;107(6):460-470.

29. Du H, Wonggom P, Tongpeth J, et al. Six-Minute Walk Test for Assessing Physical Functional Capacity in Chronic Heart Failure. Curr Heart Fail Rep. 2017;14(3):158-166.

30. Coates LA, Graham BL, Mc Fadden RG, et al. Spirometry in Primary Care. Can Respir J. 2013;20(1):13-22.

31. Ashraf M, Shaffi SA, BaHammam AS et al. Spirometry and FlowVolume Curve in Patients with Obstructive Sleep Apnea. Saudi Med J. 2008;29(2):198-202.

32. Stanchina ML, Welicky LM, Donat $\mathrm{W}$ et al. Impact of CPAP Use and Age on Mortality in Patients with Combined COPD and Obstructive Sleep Apnea: The Overlap Syndrome. J Clin Sleep Med. 2013;9(8):767-772.

33. Pedrosa RP, Maki-Nunes C, Midlej-Brito T, et al. Predictors of Obstructive Sleep Apnea in Consecutive Patients with Metabolic Syndrome. Metab Syndr Relat Disord. 2018;16(1):2-5.

34. Godoroja DD, Cioc DA. Identification of Significant Obstructive Sleep Apnoea in the Obese Patient: Development of the Novel DX-OSA Score. Rom J Anaesth Intensive Care. 2016;23(2):111-121.

35. Chousangsuntorn, Bhongmakapat T, Apirakkittikul N, et al. Computed Tomography Characterization and Comparison With Polysomnography for Obstructive Sleep Apnea Evaluation. J Oral Maxillofac Surg. 2018;76(4):854-872.

36. Young T, Skatrud J, Peppard PE. et al. Risk Factors for Obstructive Sleep Apnea in Adults. JAMA. 2004;291(16):2013-2016.

37. Davies RJ, Ali NJ, Stradling JR. et al. Neck Circumference and Other Clinical Features in the Diagnosis of the Obstructive Sleep Apnoea Syndrome. Thorax. 1992;47(2):101-105.

38. Redenius R, Murphy C, O Neill E, et al. Does CPAP Lead to Change in BMI? J Clin Sleep Med. 2008;4(3):205-209.

39. Aguiar IC, Freitas WR, Santos IR et al. Obstructive Sleep Apnea and Pulmonary Function in Patients with Severe Obesity Before and After Bariatric Surgery: A Randomized Clinical Trial. Multidiscip Respir Med. 2014;9(1):43.
40. Christopher E, Ewing GB, Burch JB, et al. Exercise Training Improves Selected Aspects of Daytime Functioning in Adults with Obstructive Sleep Apnea. J Clin Sleep Med. 2012;8(4):357-365.

41. Roberto P Martinez D, Pedroso MM, et al. Exercise, Occupational Ac tivity, and Risk of Sleep Apnea: A Cross-Sectional Study. J Clin Sleep Med. 2017;13(2):197-204.

42. Bhargava A, Guthrie JF. Unhealthy Eating Habits, Physical Exercise and Macronutrient Intakes are Predictors of Anthropometric Indicators in the Women's Health Trial: Feasibility Study in Minority Populations. Br J Nutr. 2002;88(6):719-728.

43. Plotnikoff RC, Kline CE, Youngstedt SD, et al. Effectiveness of Interventions Targeting Physical Activity, Nutrition and Healthy Weight for University and College Students: A Systematic Review and MetaAnalysis. Int J Behav Nutr Phys Act. 2015;12:45.

44. Iftikhar IH, Kline CE, Youngstedt SD, et al. Effects of Exercise Training on Sleep Apnea: A Meta-analysis. Lung. 2014;192(1):175-184.

45. Sengul YS, Ozalevli S, Oztura I, et al. The Effect of Exercise on Obstructive Sleep Apnea: A Randomized and Controlled Trial. Sleep Breath. 2011;15(1):49-56

46. Saghiv, Sira DB, Goldhammer E et al. The Effects of Aerobic and Anaerobic Exercises on Circulating Soluble-Klotho and IGF-I in Young and Elderly Adults and in CAD Patients. J Circ Biomark. 2017;6:1849454417733388.

47. Saghiv M, Circulating Soluble-Klotho and IGF-I Responses to Different Exercise Modalities in Young, Elderly and CAD Patients. J Cardiol \& Cardiovasc Ther. 2017;4(3):555637.

48. Saghiv, Chris Sherve, Ehud Goldhammer, et al. Long Lasting Chronic Resistive Training Effects on Circulating S-Klotho and IGF-1. Arch Clin Biomed Res. 2017;1(2):69-75.

49. Jonas DE. et al. Screening for Obstructive Sleep Apnea in Adults: An Evidence Review for the U.S. Preventive Services Task Force [Internet].

50. Cholidou KG, Manali ED, Kapsimalis F, et al. Heart Rate Recovery Post 6-Minute Walking Test in Obstructive Sleep Apnea: Cycle Ergometry Versus 6-Minute Walking Test in OSA Patients. Clin Res Cardiol. 2014;103(10):805-815.

51. Alameri H, Al-Kabab Y, BaHammam A, et al. Submaximal Exercise in Patients with Severe Obstructive Sleep Apnea. Sleep Breath. 2010;14(2):145-151

52. Orini M, Andrew Tinker, Patricia B, et al. Correction: Long-Term Intra-Individual Reproducibility of Heart Rate Dynamics During Exercise and Recovery in the UK Biobank Cohort. PLoS One 2018;13(2):e0193039.

53. Beitler JR, Awad KM, Bakker JP, et al. Obstructive Sleep Apnea is Associated with Impaired Exercise Capacity: A Cross-Sectional Study. $J$ Clin Sleep Med. 2014;10(11):1199-1204.

54. Hargens TA, Guill SG, Zedalis D, et al. Attenuated Heart Rate Recovery Following Exercise Testing in Overweight Young Men with Untreated Obstructive Sleep Apnea. Sleep. 2008;31(1):104-110.

55. Przybyłowski T, Bielicki P, Kumor M, et al. Exercise Capacity in Patients with Obstructive Sleep Apnea Syndrome. J Physiol Pharmacol. 2007;58 Suppl 5(Pt 2):563-574.

56. Barbosa BT, Da Cruz Santos A, Frazão M, et al. Obstructive Sleep Apnea does not Impair Cardiorespiratory Responses to Progressive Exercise Performed until Exhaustion in Hypertensive Elderly. Sleep Breath. 2018;22(2):431-437

57. Cintra F, Poyares D, Rizzi CF, et al. Cardio respiratory Response to 
Exercise in Men and Women with Obstructive Sleep Apnea. Sleep Med. 2009;10(3):368-373.

58. Shi J, Piao J, Liu B, et al. Obstructive Sleep Apnea Increases Systolic and Diastolic Blood Pressure Variability in Hypertensive Patients. Blood Press Monit. 2017;22(4):208-212.

59. Kaleth AS, Chittenden TW, Hawkins BJ, et al. Unique Cardiopulmonary Exercise Test Responses in Overweight Middle-Aged Adults with Obstructive Sleep Apnea. Sleep Med. 2007;8(2):160-168.

60. Rizzi CF, Cintra F, Mello-Fujita L, et al. Does Obstructive Sleep Apnea Impair the Cardiopulmonary Response to Exercise? Sleep. 2013;36(4):547-553

61. Vranish JR, Bailey EF. Inspiratory Muscle Training Improves Sleep and Mitigates Cardiovascular Dysfunction in Obstructive Sleep Apnea. Sleep. 2016;39(6):1179-1185.

62. Vanhecke TE, Franklin BA, Zalesin KC, et al. Cardio respiratory Fitness and Obstructive Sleep Apnea Syndrome in Morbidly Obese Patients. Chest. 2008;134(3):539-545.

63. Gries RE, Brooks LJ. Normal Oxyhemoglobin Saturation during Sleep How Low does it go?. Chest. 1996;110(6):1489-1492.

64. Min HJ, Park AY, Kim DH, et al. Neck Circumference and Lowest Oxygen Saturation Are Independently Associated with High Coexistence of Hypertension in Obstructive Sleep Apnea. Yonsei Med J. 2014;55(5):1310-1317.

65. Mieczkowski B, Ezzie ME. Update on Obstructive Sleep Apnea and its Relation to COPD. Int J Chron Obstruct Pulmon Dis. 2014;9:349-362.

66. Devon A, Papandreou C, Patil SP, et al. Diet and Exercise in the Management of Obstructive Sleep Apnoea and Cardiovascular Disease Risk. Eur Respir Rev. 2017;26(144):160110.

67. Dobrosielski DA, Patil S, Schwartz AR, et al. Effects of Exercise and Weight Loss in Older Adults with Obstructive Sleep Apnea. Med Sci Sports Exerc. 2015;47(1):20-26.

68. Lamb KL, Eston RG, Corns D. Reliability of Ratings of Perceived Exertion during Progressive Treadmill Exercise. $\mathrm{Br} J$ Sports Med. 1999;33(5):336-339.

69. Barberan-Garcia A, Arbillaga-Etxarri A, Gimeno-Santos E et al. Nordic Walking Enhances Oxygen Uptake without Increasing the Rate of Perceived Exertion in Patients with Chronic Obstructive Pulmonary Disease. Respiration. 2015;89(3):221-225.

70. Awotidebe TO, Awopeju OF, Bisiriyu LA, et al. Relationships Between Respiratory Parameters, Exercise Capacity and Psychosocial Factors in People with Chronic Obstructive Pulmonary Disease. Ann Phys Rehabil Med. 2017;60(6):387-392.

71. Al-Abed M, Behbehani K, Burk JR, et al. Cross Correlation and Scatter Plots of the Heart Rate Variability and R-Peak Envelope as Features in the Detection of Obstructive Sleep Apnea. Conf Proc IEEE Eng Med Biol Soc. 2008;2008:3488-91.

72. Loui WS, Blackshear JL, Fredrickson PA, et al. Obstructive Sleep Apnea Manifesting as Suspected Angina: Report of Three Cases. Mayo Clin Proc. 1994;69(3):244-248.

73. Tamura A, Kawano Y, Ando S, et al. Association Between Coronary Spastic Angina Pectoris and Obstructive Sleep Apnea. J Cardiol. 2010;56(2):240-244.

74. Geovanini GR, Gowdak LHW, Pereira AC, et al. OSA and Depression are Common and Independently Associated with Refractory Angina in Patients with Coronary Artery Disease. Chest. 2014;146(1):73-80.

75. Schäfer H, Koehler U, Ewig S et al. Obstructive Sleep Apnea as a Risk
Marker in Coronary Artery Disease. Cardiology. 1999;92(2):79-84.

76. Jean-Louis G, Zizi F, Brown D, et al. Obstructive Sleep Apnea and Cardiovascular Disease: Evidence and Underlying Mechanisms. Minerva Pneumol. 2009;48(4):277-293.

77. Jenkins S, Čečins N. Six-Minute Walk Test: Observed Adverse Events and Oxygen Desaturation in a Large Cohort of Patients with Chronic Lung Disease. Intern Med J. 2011;41(5):416-422.

78. Yadav YK. Exercise in the Management of Coronary Artery Disease. Med J Armed Forces India. 2007;63(4):357-361.

79. Wee Y, Burns K, Bett N, et al. Medical Management of Chronic Stable Angina. Aust Prescr. 2015;38(4):131-136.

80. Ben-Saad H, Ben Hassen I, Ghannouchi I, et al. 6-Min Walk-Test Data in Severe Obstructive-Sleep-Apnea-Hypopnea-Syndrome (OSAHS) under Continuous-Positive-Airway-Pressure (CPAP) Treatment. Respir Med. 2015;109(5):642-655.

81. Chien MY, Wu YT, Lee PL, et al. Inspiratory Muscle Dysfunction in Patients with Severe Obstructive Sleep Apnoea. Eur Respir J. 2010;35:373-380.

82. Naghan, Oldooz Aloosh, Hamze Ali Torang et al. Can the 6-Minute Walk Test Predict Severity of Obstructive Sleep Apnea Syndrome? Sleep Science and Practice. 2017;1:17.

83. O'donell, Banzett RB, Carrieri-Kohlman V, et al. Pathophysiology of Dyspnea in Chronic Obstructive Pulmonary Disease - A Roundtable. Proc Am Thorac Soc. 2007;4(2):145-168.

84. Bernhardt V, Babb TG. Exertional Dyspnoea in Obesity. Eur Respir Rev 2016;25:487-495.

85. Stendardi L, Binazzi B, Scano G. Exercise Dyspnea in Patients with COPD. Int J Chron Obstruct Pulmon Dis. 2007;2(4):429-439.

86. Cortopassi F, Castro AA, Porto EF, et al. Comprehensive Exercise Training Improves Ventilatory Muscle Function and Reduces Dyspnea Perception in Patients with COPD. Monaldi Arch Chest Dis. 2009;71(3):106-112.

87. Pan L, Guo YZ, Yan JH, et al. Does Upper Extremity Exercise Improve Dyspnea in Patients with COPD? A Meta-Analysis. Respir Med. 2012;106(11):1517-1525.

88. Pływaczewski R, Stokłosa A, Bieleń P, et al. Six-Minute Walk Test in Obstructive Sleep Apnoea. Pneumonol Alergol Pol. 2008;76(2):75-82

89. Ben-Cheikh IR, Trabelsi Y, Chouchene A et al. Changes in Six-Minute Walking Distance During Pulmonary Rehabilitation in Patients with COPD and in Healthy Subjects. Int J Chron Obstruct Pulmon Dis. 2010;5:209-215.

90. Chandra D, Wise RA, Kulkarni HS, et al. Optimizing the 6-Min Walk Test as a Measure of Exercise Capacity in COPD. Chest. 2012;142(6):1545-1552.

91. Karagullu1 H. et al. Efficacy of Pulmonary Rehabilitation Program with Cycle Ergo meter in Obstructive Sleep Apnea Syndrome. J Osteopor Phys Act.2015;3:2.

92. Giebelhaus V, Strohl KP, Lormes W, et al. Physical Exercise as an Adjunct Therapy in Sleep Apnea-An Open Trial. Sleep Breath. 2010;4:173-176.

93. Norman JF, Von Essen SG, Fuchs RH, et al. Exercise Training Effect on Obstructive Sleep Apnea Syndrome. Sleep Res Online. 2000;3(3):121129.

94. Arslan S, Erol MK, Gundogdu F, et al. Prognostic Value of 6-Minute Walk Test in Stable Outpatients with Heart Failure. Tex Heart Inst J. 2007;34(2):166-169. 
95. Ingle L. et al. The Long-Term Prognostic Significance of 6-Minute Walk Test Distance in Patients with Chronic Heart Failure. Biomed Res Int. 2014;2014:505969.

96. Dajczman E, Wardini R, Kasymjanova G, et al. Six Minute Walk Distance is a Predictor of Survival in Patients with Chronic Obstructive Pulmonary Disease Undergoing Pulmonary Rehabilitation. Can Respir J. 2015;22(4):225-229.

97. Mathia SC, Puhan MA, Lam D, et al. The Minimal Important Difference in the 6-Minute Walk Test for Patients with Pulmonary Arterial Hypertension. Am J Respir Crit Care Med. 2012;186(5):428-433.

98. Enfield K, Gammon S, Floyd J, et al. Six-Minute Walk Distance in Patients with Severe End-Stage COPD: Association with Survival after Inpatient Pulmonary Rehabilitation. $J$ Cardiopulm Rehabil Prev. 2010;30(3):195-202

99. Wise, RA, Brown CD. Minimal Clinically Important Differences in the Six-Minute Walk Test and the Incremental Shuttle Walk Test. COPD. 2005;2(1):125-129.

100. Sanseverino MA, Pecchiari M, Bona RL, et al. Limiting Factor in Walking Performance of Subjects With COPD. Respir Care. 2018;63(3):301-310

101. Mc Nicholas WT. COPD-OSA Overlap Syndrome: Evolving Evidence Regarding Epidemiology, Clinical Consequences, and Management. Chest. 2017;152(6):1318-1326

102. Zamarrón C, García Paz V, Morete E, et al. Association of Chronic Obstructive Pulmonary Disease and Obstructive Sleep Apnea Consequences. Int J Chron Obstruct Pulmon Dis. 2008;3(4):671-682.

103. Spicuzza L, Caruso D, Di Maria G, et al. Obstructive Sleep Apnoea Syndrome and its Management. Ther Adv Chronic Dis. 2015;6(5):273285

104. Freedman N. Treatment of Obstructive Sleep Apnea Syndrome. Clin Chest Med. 2010;31(2):187-201.

105. Zafari Z, Sin DD, Postma DS, et al. Individualized Prediction of LungFunction Decline in Chronic Obstructive Pulmonary Disease. CMAJ. 2016;188(14):1004-1011.

106. Checkley W, Foreman MG, Bhatt SP, et al. Differences between Absolute and Predicted Values of Forced Expiratory Volumes to Classify Ventilatory Impairment in Chronic Obstructive Pulmonary Disease. Respir Med. 2016;111:30-38.

107. Stanojevic S, Wade A, Stocks J, et al. Reference Ranges for Spirometry Across All Ages: A New Approach. Am J Respir Crit Care Med. 2008;177(3):253-260.

108. Soler X, Gaio E, Powell FL, et al. High Prevalence of Obstructive Sleep Apnea in Patients with Moderate to Severe Chronic Obstructive Pulmonary Disease. Ann Am Thorac Soc. 2015;12(8):1219-1225.

109. Wibmer T, Rüdiger $\mathrm{S}^{2}$, Kropf-Sanchen C, et al. Relation of Exercise
Capacity With Lung Volumes Before and After 6-Minute Walk Test in Subjects With COPD. Respir Care. 2014;59(11):1687-1695.

110. Zhao YY, Blackwell T, Ensrud KE, et al. Sleep Apnea and Obstructive Airway Disease in Older Men: Outcomes of Sleep Disorders in Older Men Study. Sleep. 2016;39(7):1343-1351.

111. Abdeyrim A, Yongping Zhang, Nanfang Li, et al. Impact of Obstructive Sleep Apnea on Lung Volumes and Mechanical Properties of the Respiratory System in Overweight and Obese Individuals. BMC Pulm Med. 2015;15:76.

112. Stauffer JL, White DP, Zwillich CW. Pulmonary Function in Obstructive Sleep Apnea. Relationships to Pharyngeal Resistance and CrossSectional Area. Chest. 1990;97(2):302-307.

113. Huang H, Yao L. Analysis of Airway Resistance and Hypoxemia in Overlap Syndrome. Saudi Med J. 2016;37(7):758-761.

114. Porszasz J, Emtner M, Goto S. et al. Exercise Training Decreases Ventilatory Requirements and Exercise-Induced Hyperinflation at Submaximal Intensities in Patients with COPD. Chest. 2005;128(4):2025-2034.

115. O’Donnell DE, McGuire M, Samis L et al. General Exercise Training Improves Ventilatory and Peripheral Muscle Strength and Endurance in Chronic Airflow Limitation. Am J. Resp and Criti Car Med. 1998;157(5):1489-1497.

116. Pellegrino RC, Villosio C, Milanese U, et al. Breathing during Exercise in Subjects with Mild-to-Moderate Airflow Obstruction: Effects of Physical Training. J. Appl Physiol. 1999;87(5):1697-1704.

117. Gigliotti FC, Coli C, Bianchi R, et al. Exercise Training Improves Exertional Dyspnea in Patients with COPD: Evidence of the Role of Mechanical Factors. Chest. 2003;123(6):1794-1802.

118. Johnson $\mathrm{BD}$, Beck $\mathrm{KC}$, Olson LJ, et al. Ventilatory Constraints during Exercise in Patients with Chronic Heart Failure. Chest 2000;117(2):321-332.

119. O’Donnell DE, Guenette JA, Maltais F, et al. Decline of Resting Inspiratory Capacity in COPD: The Impact on Breathing Pattern, Dyspnea, and Ventilatory Capacity during Exercise. Chest. 2012;141(3):753-762.

120. Swanney MP, Ruppel G, Enright PL, et al. Using the Lower Limit of Normal for the FEV1/FVC Ratio Reduces the Misclassification of Airway Obstruction. Thorax. 2008;63(12):1046-1051.

121. Cheng YJ, Macera CA, Addy CL, et al. Effects of Physical Activity on Exercise Tests and Respiratory Function. Br J Sports Med. 2003;37:521-528.

122. Sahebjami H, Gartside PS. Pulmonary Function in Obese Subjects with a Normal FEV1/FVC Ratio. Chest. 1996;110(6):1425-1429.

123. Zammit C, Liddicoat H, Moonsie I, et al. Obesity and Respiratory Diseases. Int J Gen Med. 2010;3:335-343. 